\title{
Breathers and Rogue Waves On The Double- Periodic Background for The Reverse-Space-Time Derivative Nonlinear Schrödinger Equation
}

\section{Huijuan Zhou}

East China Normal University

Yong Chen ( $\sim$ profchenyong@163.com )

East China Normal University https://orcid.org/0000-0002-6008-6542

\section{Research Article}

Keywords: Reverse-space-time derivative nonlinear Schrödinger equation, Generalized Darboux transformation, Breathers and rogue waves on the double-periodic background

Posted Date: July 6th, 2021

DOI: https://doi.org/10.21203/rs.3.rs-658176/v1

License: (c) (1) This work is licensed under a Creative Commons Attribution 4.0 International License.

Read Full License

Version of Record: A version of this preprint was published at Nonlinear Dynamics on October 13th, 2021. See the published version at https://doi.org/10.1007/s11071-021-06953-8. 


\title{
Breathers and rogue waves on the double-periodic background for the reverse-space-time derivative nonlinear Schrödinger equation
}

\author{
Huijuan Zhou · Yong Chen
}

Received: date / Accepted: date

\begin{abstract}
The dynamic behaviors of the solutions for the reverse-space-time derivative nonlinear Schrödinger equation are studied by Darboux transformation. The breathers on the periodic and double-periodic background are derived by the $N$-fold Darboux transformation. The rogue waves on the periodic and double-periodic background are constructed by the generalized Darboux transformation. It is worth mentioning that the breathers and rogue waves on double-periodic background based on the plane wave seed solution are first constructed. The two peak, four peak rogue waves on the double-periodic background are found. And the rogue waves on the double-periodic background can be transformed into the classical rogue wave on the plane wave background with a special reduction relation.
\end{abstract}

Keywords Reverse-space-time derivative nonlinear Schrödinger equation · Generalized Darboux transformation · Breathers and rogue waves on the double-periodic background

PACS 02.30.Ik $\cdot 02.30 . J r \cdot 05.45 . \mathrm{Yv}$

The project is supported by Global Change Research Program of China (No.2015CB953904), National Natural Science Foundation of China (No.11675054) and Science and Technology Commission of Shanghai Municipality (No.18dz2271000).

Huijuan Zhou

School of Mathematical Sciences, Shanghai Key Laboratory of PMMP, East China Normal University, Shanghai, 200241, P.R. China

Yong Chen ( $\bigotimes_{\text {corresponding author) }}$

School of Mathematical Sciences, Shanghai Key Laboratory of PMMP, East China Normal University, Shanghai, 200241, P.R. China

College of Mathematics and Systems Science, Shandong University of Science and Technology, Qingdao, 266590, P.R. China

Department of Physics, Zhejiang Normal University, Jinhua, 321004, P.R. China

Tel.: +86-21-62224199

Fax: +86-21-62235025

E-mail: profchenyong@163.com 


\section{Introduction}

Nonlinear evolution equations play an important role and their solutions have been a hot research spot, including soliton [1,2,3,4, breather [5,6,7, rogue wave [8,9,10,11] and others [12, 13, 14, 15. The derivative nonlinear Schrödinger equation (DNLS) [16, 17, 18, 19]

$$
i q_{t}-q_{x x}+i\left(q^{2} q^{*}\right)_{x}=0,
$$

where the complex function $q=q(x, t)$ denotes the wave envelopes, ${ }^{*}$ denotes the complex conjugation. Eq.(1) arises in the study of circular polarized Alfvén waves in plasma [20], propagating parallel to the magnetic field [21], which is one of the most important integrable systems in mathematics and physics. Recently, the equation is also used to describe large-amplitude magnetohydrodynamic waves [22,23] of plasmas, nonlinear optics, the sub-picosecond and femtosecond pulses in single-mode optical fiber [24,25,26]. The DNLS and nonlocal DNLS equations are reduced from the Kaup-Newell system [27,28 and are Lax integrable. There generate many new physical phenomena and have important physical significance when nonlocal terms are added to nonlinear equations.

There have been some researches on nonlocal DNLS equations in recent years. For example, in the literature [29], the global bounded solutions of the nonlocal DNLS equation have been obtained from zero seed solution by Darboux transformation (DT) 30,31, 32, 33, 34, 35, . All achievable nonlocal equation of the DNLS equations have been derived in 36. In 37, the periodic bounded solutions of the second-type nonlocal DNLS equation from zero seed solutions have been studied. The reverse-space-time DNLS equation [38] is as follows

$$
i q_{t}-q_{x x}+i\left(q^{2} q(-x,-t)\right)_{x}=0,
$$

which is one type of the nonlocal DNLS equation.

In general, it is extremely nontrivial to construct the rogue waves on a periodic background, which is usually associated with complicated Jacobi elliptic functions [39,40,41, PT symmetry [42, integrable equations with variable coefficients [4],44], or vector integrable equations [45]. In 46. 47, the rogue waves on the periodic background are constructed by generalized DT of odd order. The rogue waves on the double-periodic background in the hydrodynamical experiments are possible due to the rogue waves on the continuous wave background were observed in laser optics and water tanks [48. It is of great significance to research the rogue waves on the doubleperiodic background.

In this work, we construct breathers on periodic and double-periodic background for the reverse-space-time DNLS equation by $N$-fold DT. Based on the DT of determinant form, the odd and even order generalized DT are constructed by Taylor expansion. Higher order rogue waves on periodic background are generated by the odd-order generalized DT, and higher order rogue waves on double-periodic background are constructed by even order generalized DT. By taking the dynamics analysis of the rogue waves on double-periodic background, we show two types of structure: the two peak and four peak. And the rogue waves on the double-periodic background can be transformed into the classical rogue wave on the plane wave background. This is an effective method to construct the breather and rogue waves on double-periodic background without using Jacobi elliptic functions $[49,50,51,52,53$.

The organizational structure of this paper is as follows. In section 2, the Lax pair of Eq.(2) is obtained by the Kaup-Newell system at the special reduction relation. And then we give the detailed derivation of the one-fold DT formula. Furthermore, the determinant representation of the $N$-fold DT formula is given. In section 3, the symmetric relation of the eigenfunctions are deduced. And then the periodic wave solution, bright soliton solution, dark soliton solution, 
breather solution, rogue waves, double-periodic solution, breathers on the periodic and doubleperiodic background are given from zero seed and non-zero seed solutions. In section 4, we construct rogue waves on periodic background and double-periodic background by generalized DT formula. At the same time, the dynamic behaviors of the solutions are given. The final section is devoted to conclusion.

\section{DT of the reverse-space-time DNLS equation}

\subsection{Lax pair of the reverse-space-time DNLS equation}

Starting from the Kaup-Newell system [54,55, different Lax pairs can be obtained by different reductions. When $r(x, t)=-q^{*}(x, t)$, the Lax pair of DNLS equation can be get. When the reduction condition is $r(x, t)=-q(-x,-t)$, the Lax pair of the reverse-space-time DNLS equation can be obtained as follows.

$$
\begin{gathered}
\Psi_{x}=\left(i \sigma \lambda^{2}+Q \lambda\right) \Psi=U \Psi \\
\Psi_{t}=\left(2 i \sigma \lambda^{4}+V_{3} \lambda^{3}+V_{2} \lambda^{2}+V_{1} \lambda\right) \Psi=V \Psi,
\end{gathered}
$$

with

$$
\Psi=\left(\begin{array}{l}
\phi \\
\varphi
\end{array}\right), \quad \sigma=\left(\begin{array}{cc}
1 & 0 \\
0 & -1
\end{array}\right), \quad Q=\left(\begin{array}{cc}
0 & q \\
-q(-x,-t) & 0
\end{array}\right)
$$

$$
V_{3}=2 Q, \quad V_{2}=i Q^{2}, \quad V_{1}=Q^{3}+i Q_{x} \sigma=\left(\begin{array}{c}
0 \\
i q_{x}(-x,-t)+q(-x,-t)^{2} q
\end{array} \begin{array}{c}
-i q_{x}-q^{2} q(-x,-t) \\
0
\end{array}\right) .
$$

The spectral parameter $\lambda$ is an arbitrary complex number, $\Psi$ is the eigenfunction corresponds to $\lambda$. Eq. (2) can be derived from the integrable condition $U_{t}-V_{x}+[U, V]=0$ of (3) and (4).

\subsection{One-fold DT of the reverse-space-time DNLS equation}

DT has unique advantages in constructing solutions due to pure algebraic construction. This section, the DT for the Eq. 22 will be introduced. Under gauge transformation

$$
\Psi^{[1]}=T \Psi,
$$

the spectral problem (3) and (4) can transform to

$$
\begin{aligned}
& \Psi_{x}^{[1]}=\left(i \sigma \lambda^{2}+Q^{[1]} \lambda\right) \Psi^{[1]}=U^{[1]} \Psi^{[1]} \\
& \Psi_{t}^{[1]}=\left(2 i \sigma \lambda^{4}+V_{3}^{[1]} \lambda^{3}+V_{2}^{[1]} \lambda^{2}+V_{1}^{[1]} \lambda\right) \Psi^{[1]}=V^{[1]} \Psi^{[1]}
\end{aligned}
$$

Where

$$
\begin{aligned}
& Q^{[1]}=\left(\begin{array}{cc}
0 & q[1] \\
-q[1](-x,-t) & 0
\end{array}\right) \\
& V_{3}^{[1]}=2 q[1], \quad V_{2}^{[1]}=i Q^{[1] 2}, \\
& V_{1}^{[1]}=Q^{[1] 3}+i q[1]_{x} \sigma=\left(\begin{array}{c}
0 \\
i q[1]_{x}(-x,-t)+q[1]^{2}(-x,-t) q[1]
\end{array} \begin{array}{c}
-i q[1]_{x}-q[1]^{2} q[1](-x,-t) \\
0
\end{array}\right) .
\end{aligned}
$$

After derivation, get the following conclusion

$$
T_{x}=U^{[1]} T-T U,
$$




$$
T_{t}=V^{[1]} T-T V .
$$

Further

$$
U^{[1]}=T_{x} T^{-1}+T U T^{-1}, \quad V^{[1]}=T_{t} T^{-1}+T V T^{-1} .
$$

Then, the following identity can be deduced

$$
U_{t}^{[1]}-V_{x}^{[1]}+\left[U^{[1]}, V^{[1]}\right]=T\left(U_{t}-V_{x}+[U, V]\right) T^{-1} .
$$

Due to the matrix $T$ is nonsingular, the zero curvature equation $U_{t}-V_{x}+[U, V]=0$ is equivalent to $U_{t}^{[1]}-V_{x}^{[1]}+\left[U^{[1]}, V^{[1]}\right]=0$. This implies that, in order to make spectral problem Eq. (3) invariant under the gauge transformation Eq.(5), it is crucial to find a matrix $T$ so that $U^{[1}$, $V^{[1]}$ have the same forms as $U, V$. At the same time, the old solution $(q, q(-x,-t))$ in spectral matrixes $U, V$ are mapped into new solution $(q[1], q[1](-x,-t))$ in transformed spectral matrixes $U^{[1]}, V^{[1]}$.

In general, if the Darboux matrix $T$ is a polynomial of the parameter $\lambda$, for simple, take $T$ as

$$
T=\left(\begin{array}{ll}
a_{1} & b_{1} \\
c_{1} & d_{1}
\end{array}\right) \lambda+\left(\begin{array}{ll}
a_{0} & b_{0} \\
c_{0} & d_{0}
\end{array}\right) .
$$

Substitute the Darboux matrix $T$ into Eq.(7), by comparing the coefficient in terms of $\lambda^{i}$ $\lambda^{3}:$

$$
b_{1}=c_{1}=0,
$$

$\lambda^{2}:$

$$
-q[1] d_{1}+a_{1} q-2 i b_{0}=2 i c_{0}-d_{1} q(-x,-t)+q[1](-x,-t) a_{1}=0,
$$

$\lambda:$

$$
\begin{aligned}
& -q[1] c_{0}-b_{0} q(-x,-t)+a_{1 x}=-q[1] d_{0}+a_{0} q=0 \\
& -d_{0} q(-x,-t)+q[1](-x,-t) a_{0}=c_{0} q+q[1](-x,-t) b_{0}+d_{1 x}=0
\end{aligned}
$$

$\lambda^{0}:$

$$
a_{0 x}=b_{0 x}=c_{0 x}=d_{0 x}=0 .
$$

In the same way, substitute the Darboux matrix $T$ into Eq. 8 , by comparing the coefficient in terms of $\lambda^{i}$ $\lambda^{4}:$

$$
-2 i b_{0}+a_{1} q-q[1] d_{1}=2 i c_{0}-d_{1} q(-x,-t)+q[1](-x,-t) a_{1}=0
$$

$\lambda^{3}:$

$$
\begin{aligned}
& i q[1](-x,-t) q[1] a_{1}-i a_{1} q q(-x,-t)-2 b_{0} q(-x,-t)-2 q[1] c_{0} \\
& \quad=2 a_{0} q-2 q[1] d_{0}=-2 d_{0} q(-x,-t)+2 q[1](-x,-t) a_{0} \\
& =i d_{1} q q(-x,-t)-i q[1](-x,-t) q_{1} d_{1}+2 c_{0} q+2 q[1](-x, t) b_{0}=0
\end{aligned}
$$

$\lambda^{2}:$

$$
\begin{aligned}
& i q[1](-x,-t) q[1] a_{0}-i a_{0} q q(-x,-t)=i b_{0} q q(-x,-t)-i q_{x} a_{1}-a_{1} q^{2} q(-x,-t) \\
& +i q[1](-x,-t) q[1] b_{0}+q[1](-x,-t) q[1]^{2} d_{1}+i d_{1} q[1]_{x}=i d_{1} q_{x}(-x,-t) \\
& -i c_{0} q q(-x,-t)+d_{1} q^{2}(-x,-t) q-i q[1]_{x}(-x,-t) a_{1}-q[1]^{2}(-x,-t) q[1] a_{1} \\
& -i q[1](-x,-t) q[1] c_{0}=i d_{0} q q(-x,-t)-i q[1](-x,-t) q[1] d_{0}=0
\end{aligned}
$$


$\lambda:$

$$
\begin{aligned}
& i b_{0} q_{x}(-x,-t)+b_{0} q^{2}(-x,-t) q+i c_{0} q[1]_{x}+q[1](-x,-t) q[1]^{2} c_{0}+a_{1 t} \\
& =-i a_{0} q_{x}-a_{0} q^{2} q(-x,-t)+q[1](-x,-t) q[1]^{2} d_{0}+i q[1]_{x} d_{0} \\
& =i d_{0} q_{x}(-x,-t)+d_{0} q^{2}(-x,-t) q-i a_{0} q[1]_{x}(-x,-t)-a_{0} q[1]^{2}(-x,-t) q[1] \\
& =-i q_{x} c_{0}-q^{2} q(-x,-t) c_{0}-i q[1]_{x}(-x,-t) b_{0}-q[1]^{2}(-x,-t) q[1] b_{0}+d_{1 t}
\end{aligned}
$$

$\lambda^{0}:$

$$
a_{0 t}=b_{0 t}=c_{0 t}=d_{0 t}=0 .
$$

So $a_{0}, b_{0}, c_{0}, d_{0}$ are constants, $a_{1}, d_{1}$ are undetermined functions about $x$ and $t$. And the new solution $q[1]$ can be shown in the following DT formula which is obtained by the Eq.11]

$$
\left\{\begin{array}{l}
q[1]=\frac{a_{1}}{d_{1}} q-2 i \frac{b_{0}}{d_{1}}, \\
q[1](-x,-t)=\frac{d_{1}}{a_{1}} q(-x,-t)-2 i \frac{c_{0}}{a_{1}} .
\end{array}\right.
$$

In order to obtain the specific expression of the elements in the DT matrix $T$, for simplicity, let $a_{0}=d_{0}=0$ then

$$
T_{1}=\left(\begin{array}{cc}
a_{1} & 0 \\
0 & d_{1}
\end{array}\right) \lambda+\left(\begin{array}{cc}
0 & b_{0} \\
c_{0} & 0
\end{array}\right) .
$$

Next, we will represent $a_{1}, d_{1}$ by the eigenfunction representation. First, introduce the eigenfunction $\Psi_{j}$ corresponding to $\lambda_{j}$ as below in the spectral problem Eq. (3) and Eq.(4)

$$
\Psi_{j}=\left(\begin{array}{c}
\phi_{j} \\
\varphi_{j}
\end{array}\right), \quad j=1,2, \ldots n, \quad \phi_{j}=\phi_{j}\left(x, t, \lambda_{j}\right), \quad \varphi_{j}=\varphi_{j}\left(x, t, \lambda_{j}\right) .
$$

In the following theorem, the one-fold DT formula is given by the eigenfunction of the spectral problem Eq. (3) and Eq. (4).

Theorem 1 Let $b_{0}=c_{0}=\lambda_{1}$, the one-fold DT formula is given by the eigenfunction $\Psi_{1}$ associated with $\lambda_{1}$ as follows

$$
\begin{aligned}
& q[1]=\left(\frac{\varphi_{1}}{\phi_{1}}\right)^{2} q+2 i \frac{\varphi_{1}}{\phi_{1}} \lambda_{1}, \\
& q[1](-x,-t)=\left(\frac{\phi_{1}}{\varphi_{1}}\right)^{2} q(-x,-t)+2 i \frac{\phi_{1}}{\varphi_{1}} \lambda_{1} .
\end{aligned}
$$

The elements of one-fold DT matrix are parameterized as below

$$
T_{1}\left(\lambda ; \lambda_{1}\right)=\left(\begin{array}{cc}
-\lambda \frac{\varphi_{1}}{\phi_{1}} & \lambda_{1} \\
\lambda_{1} & -\lambda \frac{\phi_{1}}{\varphi_{1}}
\end{array}\right) .
$$

And then the new eigenfunction $\Psi_{j}^{[1]}$ corresponding to $\lambda_{j}$ is

$$
\Psi_{j}^{[1]}=\left(\begin{array}{c}
\frac{1}{\phi_{1}}\left|\begin{array}{ll}
-\lambda_{j} \phi_{j} & \varphi_{j} \\
-\lambda_{1} \phi_{1} & \varphi_{1}
\end{array}\right| \\
\frac{1}{\varphi_{1}}\left|\begin{array}{ll}
-\lambda_{j} \varphi_{j} & \phi_{j} \\
-\lambda_{1} \varphi_{1} & \phi_{1}
\end{array}\right|
\end{array}\right) .
$$


Proof The identity $\left(a_{1} d_{1}\right)_{x}=0$ can be derived by the Eq.(11) and Eq. 12. Without loss of generality, taking $a_{1}=\frac{1}{d_{1}}$ in the following. Let $b_{0}=c_{0}=\lambda_{1}$, due to the kernel problem of Darboux matrix

$$
\left.T_{1}\left(\lambda ; \lambda_{1}\right)\right|_{\lambda=\lambda_{1}} \Psi_{1}=\left(\begin{array}{cc}
a_{1} \lambda_{1} & \lambda_{1} \\
\lambda_{1} & d_{1} \lambda_{1}
\end{array}\right)\left(\begin{array}{l}
\phi_{1} \\
\varphi_{1}
\end{array}\right)=0,
$$

then $a_{1}=-\frac{\varphi_{1}}{\phi_{1}}, \quad d_{1}=-\frac{\phi_{1}}{\varphi_{1}}$. Substituting the result into Eq.13 and Eq.114, the Eq. 15 and Eq.16] can be derived. Further, $\Psi_{j}^{[1]}$ is given as follows by the Eq.16.

$$
\Psi_{j}^{[1]}=\left.T_{1}\left(\lambda ; \lambda_{1}\right)\right|_{\lambda=\lambda_{j}} \Psi_{j}=\left.\left(\begin{array}{cc}
-\lambda \frac{\varphi_{1}}{\phi_{1}} & \lambda_{1} \\
\lambda_{1} & -\lambda \frac{\phi_{1}}{\varphi_{1}}
\end{array}\right)\right|_{\lambda=\lambda_{j}}\left(\begin{array}{c}
\phi_{j} \\
\varphi_{j}
\end{array}\right)=\left(\begin{array}{c}
\frac{1}{\phi_{1}}\left|\begin{array}{cc}
-\lambda_{j} \phi_{j} & \varphi_{j} \\
-\lambda_{1} \phi_{1} & \varphi_{1}
\end{array}\right| \\
\frac{1}{\varphi_{1}}\left|\begin{array}{ll}
-\lambda_{j} \varphi_{j} & \phi_{j} \\
-\lambda_{1} \varphi_{1} & \phi_{1}
\end{array}\right|
\end{array}\right) .
$$

\section{3 $N$-fold DT of reverse-space-time DNLS equation}

In the previous section, the concrete form of the one-fold Darboux matrix has been given. In this section, we will establish the determinant representation of the $N$-fold DT for Eq. 22. The spectral problem (6) can transform to

$$
\begin{aligned}
& \Psi_{x}^{[2]}=U^{[2]} \Psi^{[2]}, \\
& \Psi_{t}^{[2]}=V^{[2]} \Psi^{[2]},
\end{aligned}
$$

under the gauge transformation

$$
\Psi^{[2]}=T^{[2]} \Psi^{[1]}=T^{[2]} T_{1} \Psi=T_{2} \Psi .
$$

According to the form of $T_{1}$ in Eq.(14), where

$$
T^{[2]}=\left(\begin{array}{cc}
a_{[2]} & 0 \\
0 & d_{[2]}
\end{array}\right) \lambda+\left(\begin{array}{cc}
0 & b_{[1]} \\
c_{[1]} & 0
\end{array}\right) .
$$

Then

$$
T_{2}=T^{[2]} T_{1}=\left(\begin{array}{cc}
a_{[2]} a_{1} & 0 \\
0 & d_{[2]} d_{1}
\end{array}\right) \lambda^{2}+\left(\begin{array}{cc}
0 & a_{[2]} b_{0}+b_{[1]} d_{1} \\
c_{[1]} a_{1}+d_{[2]} c_{0} & 0
\end{array}\right) \lambda+\left(\begin{array}{cc}
b_{[1]} c_{0} & 0 \\
0 & c_{[1]} b_{0}
\end{array}\right) .
$$

Repeat the iterative process above,

$$
\Psi^{[3]}=T^{[3]} \Psi^{[3]}=T^{[3]} T^{[2]} T_{1} \Psi=T_{3} \Psi,
$$

where

$$
T^{[3]}=\left(\begin{array}{cc}
a_{[3]} & 0 \\
0 & d_{[3]}
\end{array}\right) \lambda+\left(\begin{array}{cc}
0 & b_{[2]} \\
c_{[2]} & 0
\end{array}\right) .
$$

Then the three-fold Darboux matrix can be given as

$$
\begin{aligned}
T_{3} & =T^{[3]} T^{[2]} T_{1}=\left(\begin{array}{cc}
a_{[3]} a_{[2]} a_{1} & 0 \\
0 & d_{[3]} d_{[2]} d_{1}
\end{array}\right) \lambda^{3} \\
& +\left(\begin{array}{cc}
0 & a_{[3]} a_{[2]} b_{0}+a_{[3]} b_{[1]} d_{1}+b_{[2]} d_{[2]} d_{1} \\
c_{[2]} a_{[2]} a_{1}+d_{[3]} c_{[1]} a_{1}+d_{[3]} d_{[2]} c_{0} & 0
\end{array}\right) \lambda^{2} \\
& +\left(\begin{array}{cc}
a_{[3]} b_{[1]} c_{0}+b_{[2]} c_{[1]} a_{1}+b_{[2]} d_{[2]} c_{0} & 0 \\
0 & c_{[2]} a_{[2]} b_{0}+c_{[2]} b_{[1]} d_{1}+d_{[3]} c_{[1]} b_{0}
\end{array}\right) \lambda \\
& +\left(\begin{array}{cc}
0 & b_{[2]} c_{[1]} b_{0} \\
c_{[2]} b_{[1]} c_{0} & 0
\end{array}\right) .
\end{aligned}
$$


After $n$ times iterations, the form of $N$-fold Darboux matrix is as follows

$$
T_{n}=T_{n}\left(\lambda ; \lambda_{1}, \lambda_{2}, \cdots, \lambda_{n}\right)=\sum_{i=0}^{n} P_{i} \lambda^{i}
$$

where $P_{i}(1 \leq i \leq n)$ is the matrix function of $x$ and $t$.

$$
\begin{gathered}
P_{n}=\left\{\left(\begin{array}{cc}
a_{n} & 0 \\
0 & d_{n}
\end{array}\right) \mid a_{n}, d_{n} \text { are complex functions of } x \text { and } t\right\}, \\
P_{n-1}=\left\{\left(\begin{array}{cc}
0 & b_{n-1} \\
c_{n-1} & 0
\end{array}\right) \mid b_{n-1}, c_{n-1} \text { are complex functions of } x \text { and } t\right\} .
\end{gathered}
$$

Here $P_{0}$ is a constant matrix,

$$
P_{0}=\left\{\begin{array}{l}
\left(\begin{array}{cc}
a_{0} & 0 \\
0 & d_{0}
\end{array}\right), \text { when } n \text { is even; } \\
\left(\begin{array}{cc}
0 & b_{0} \\
c_{0} & 0
\end{array}\right), \text { when } n \text { is odd. }
\end{array}\right.
$$

According to the parity of $n$, which leads to the separate discussion on the kernel problem of DT matrix T.

$$
\left.T_{n}\left(\lambda ; \lambda_{1}, \cdots, \lambda_{n}\right)\right|_{\lambda=\lambda_{k}} \Psi_{k}=\sum_{i=0}^{n} P_{i} \lambda_{k}^{i} \Psi_{k}=0, k=1,2, \cdots, n
$$

coefficients $P_{i}$ can be solved by Cramer's rule. Thus the determinant representation of the $T_{n}$ is given in the following theorem.

Theorem 2 When $n$ is even, i.e. $n=2 k, k=1,2,3, \cdots$. The $N$-fold DT matrix $T_{n}$ can be expressed as

$$
T_{n}=T_{n}\left(\lambda ; \lambda_{1}, \lambda_{2}, \cdots, \lambda_{n}\right)=\left(\begin{array}{cc}
\frac{\left(\widehat{T}_{n}\right)_{11}}{M_{n}} & \frac{\left(\widehat{T}_{n}\right)_{12}}{M_{n}} \\
\frac{\left(\widehat{T}_{n}\right)_{21}}{\widehat{M}_{n}} & \frac{\left(\widehat{T}_{n}\right)_{22}}{\widehat{M}_{n}}
\end{array}\right)
$$

with

$$
\begin{aligned}
& M_{n}=\left|\begin{array}{ccccccc}
\lambda_{1}^{n} \phi_{1} & \lambda_{1}^{n-1} \varphi_{1} & \lambda_{1}^{n-2} \phi_{1} & \lambda_{1}^{n-3} \varphi_{1} & \ldots & \lambda_{1}^{2} \phi_{1} & \lambda_{1} \varphi_{1} \\
\lambda_{2}^{n} \phi_{2} & \lambda_{2}^{n-1} \varphi_{2} & \lambda_{2}^{n-2} \phi_{2} & \lambda_{2}^{n-3} \varphi_{2} & \ldots & \lambda_{2}^{2} \phi_{2} & \lambda_{2} \varphi_{2} \\
\vdots & \vdots & \vdots & \vdots & \vdots & \vdots & \vdots \\
\lambda_{n}^{n} \phi_{n} & \lambda_{n}^{n-1} \varphi_{n} & \lambda_{n}^{n-2} \phi_{n} & \lambda_{n}^{n-3} \varphi_{n} & \ldots & \lambda_{n}^{2} \phi_{n} & \lambda_{n} \varphi_{n}
\end{array}\right| \\
& \widehat{M}_{n}=\left|\begin{array}{ccccccc}
\lambda_{1}^{n} \varphi_{1} & \lambda_{1}^{n-1} \phi_{1} & \lambda_{1}^{n-2} \varphi_{1} & \lambda_{1}^{n-3} \phi_{1} & \ldots & \lambda_{1}^{2} \varphi_{1} & \lambda_{1} \phi_{1} \\
\lambda_{2}^{n} \varphi_{2} & \lambda_{2}^{n-1} \phi_{2} & \lambda_{2}^{n-2} \varphi_{2} & \lambda_{2}^{n-3} \phi_{2} & \ldots & \lambda_{2}^{2} \varphi_{2} & \lambda_{2} \phi_{2} \\
\vdots & \vdots & \vdots & \vdots & \vdots & \vdots & \vdots \\
\lambda_{n}^{n} \varphi_{n} & \lambda_{n}^{n-1} \phi_{n} & \lambda_{n}^{n-2} \varphi_{n} & \lambda_{n}^{n-3} \phi_{n} & \ldots & \lambda_{n}^{2} \varphi_{n} & \lambda_{n} \phi_{n}
\end{array}\right|,
\end{aligned}
$$

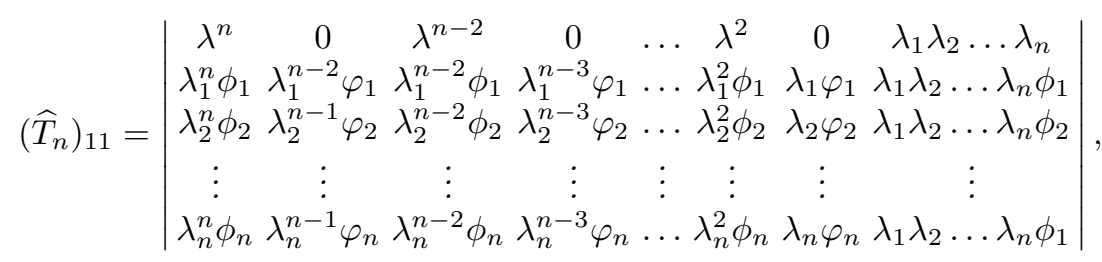




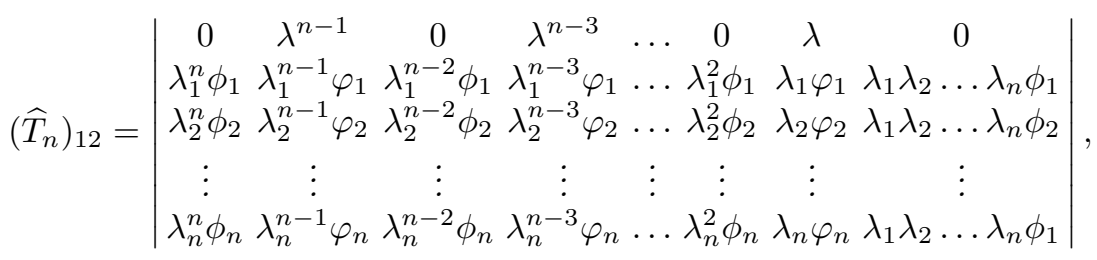

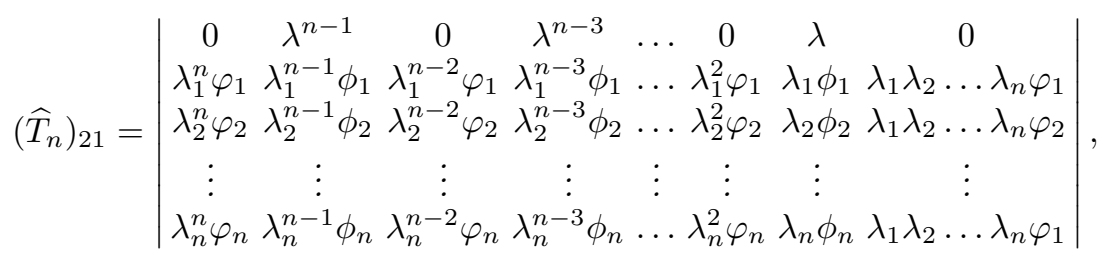

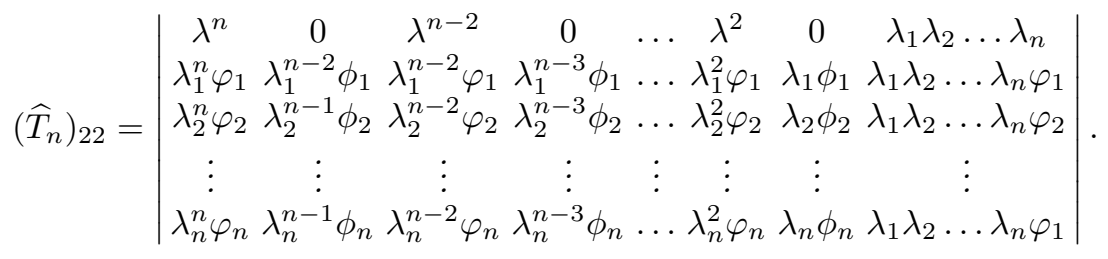

When $n$ is odd, i.e. $n=2 k+1, k=1,2,3, \cdots$. The $N$-fold DT matrix $T_{n}$ can be expressed as

$$
T_{n}=T_{n}\left(\lambda ; \lambda_{1}, \lambda_{2}, \cdots, \lambda_{n}\right)=\left(\begin{array}{ll}
\frac{\left(\widetilde{T}_{n}\right)_{11}}{\underline{Y}_{n}} & \frac{\left(\widetilde{T}_{n}\right)_{12}}{Y_{n}} \\
\frac{\left(\widetilde{T}_{n}\right)_{21}}{\widetilde{Y}_{n}} & \frac{\left(\widetilde{T}_{n}\right)_{22}}{\widetilde{Y}_{n}}
\end{array}\right),
$$

with

$$
\begin{aligned}
& Y_{n}=\left|\begin{array}{ccccccc}
\lambda_{1}^{n} \phi_{1} & \lambda_{1}^{n-1} \varphi_{1} & \lambda_{1}^{n-2} \phi_{1} & \lambda_{1}^{n-3} \varphi_{1} & \ldots & \lambda_{1}^{2} \varphi_{1} & \lambda_{1} \phi_{1} \\
\lambda_{2}^{n} \phi_{2} & \lambda_{2}^{n-1} \varphi_{2} & \lambda_{2}^{n-2} \phi_{2} & \lambda_{2}^{n-3} \varphi_{2} & \ldots & \lambda_{2}^{2} \varphi_{2} & \lambda_{2} \phi_{2} \\
\vdots & \vdots & \vdots & \vdots & \vdots & \vdots & \vdots \\
\lambda_{n}^{n} \phi_{n} & \lambda_{n}^{n-1} \varphi_{n} & \lambda_{n}^{n-2} \phi_{n} & \lambda_{n}^{n-3} \varphi_{n} & \ldots & \lambda_{n}^{2} \varphi_{n} & \lambda_{n} \phi_{n}
\end{array}\right| \\
& \widetilde{Y}_{n}=\left|\begin{array}{ccccccc}
\lambda_{1}^{n} \varphi_{1} & \lambda_{1}^{n-1} \phi_{1} & \lambda_{1}^{n-2} \varphi_{1} & \lambda_{1}^{n-3} \phi_{1} & \ldots & \lambda_{1}^{2} \phi_{1} & \lambda_{1} \varphi_{1} \\
\lambda_{2}^{n} \varphi_{2} & \lambda_{2}^{n-1} \phi_{2} & \lambda_{2}^{n-2} \varphi_{2} & \lambda_{2}^{n-3} \phi_{2} & \ldots & \lambda_{2}^{2} \phi_{2} & \lambda_{2} \varphi_{2} \\
\vdots & \vdots & \vdots & \vdots & \vdots & \vdots & \vdots \\
\lambda_{n}^{n} \varphi_{n} & \lambda_{n}^{n-1} \phi_{n} & \lambda_{n}^{n-2} \varphi_{n} & \lambda_{n}^{n-3} \phi_{n} & \ldots & \lambda_{n}^{2} \phi_{n} & \lambda_{n} \varphi_{n}
\end{array}\right| \\
& \left(\widetilde{T}_{n}\right)_{11}=\left|\begin{array}{cccccccc}
\lambda^{n} & 0 & \lambda^{n-2} & 0 & \ldots & \lambda^{2} & 0 & \lambda_{1} \lambda_{2} \ldots \lambda_{n} \\
\lambda_{1}^{n} \phi_{1} & \lambda_{1}^{n-2} \varphi_{1} & \lambda_{1}^{n-2} \phi_{1} & \lambda_{1}^{n-3} \varphi_{1} & \ldots & \lambda_{1}^{2} \phi_{1} & \lambda_{1} \varphi_{1} & \lambda_{1} \lambda_{2} \ldots \lambda_{n} \phi_{1} \\
\lambda_{2}^{n} \phi_{2} & \lambda_{2}^{n-1} \varphi_{2} & \lambda_{2}^{n-2} \phi_{2} & \lambda_{2}^{n-3} \varphi_{2} & \ldots & \lambda_{2}^{2} \phi_{2} & \lambda_{2} \varphi_{2} & \lambda_{1} \lambda_{2} \ldots \lambda_{n} \phi_{2} \\
\vdots & \vdots & \vdots & \vdots & \vdots & \vdots & \vdots & \vdots \\
\lambda_{n}^{n} \phi_{n} & \lambda_{n}^{n-1} \varphi_{n} & \lambda_{n}^{n-2} \phi_{n} & \lambda_{n}^{n-3} \varphi_{n} & \ldots & \lambda_{n}^{2} \phi_{n} & \lambda_{n} \varphi_{n} & \lambda_{1} \lambda_{2} \ldots \lambda_{n} \phi_{1}
\end{array}\right|,
\end{aligned}
$$

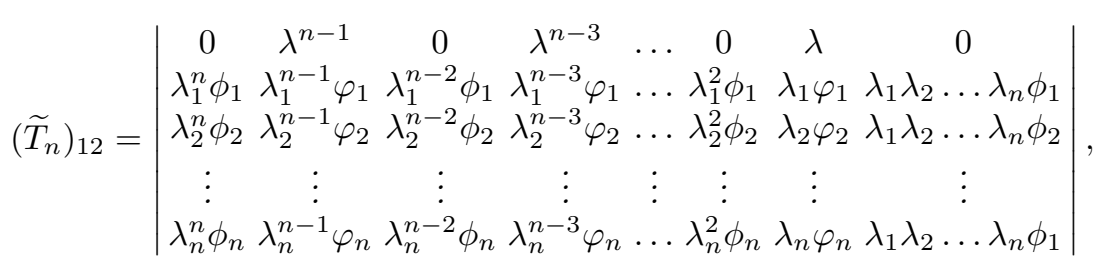




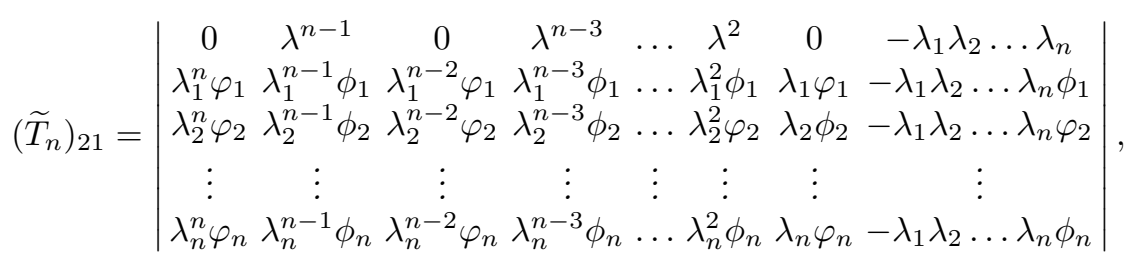

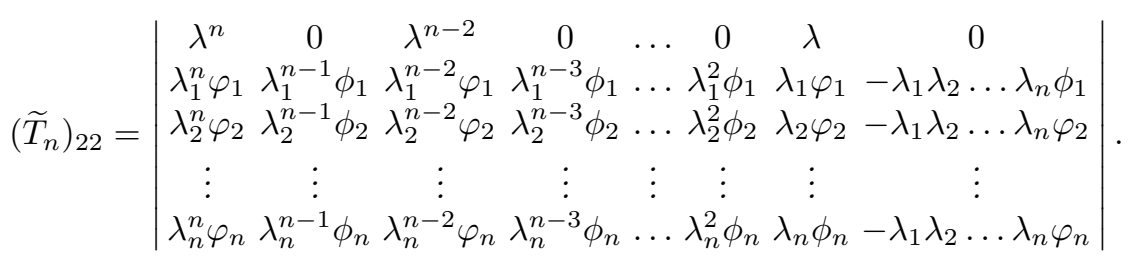

Next, we research the determinant representation of the new solutions $(q[n], q[n](-x,-t))$. Under covariant requirement of spectral problem Eq.(3) and Eq.(4),

$$
\begin{gathered}
\Psi_{x}^{[n]}=\left(i \sigma \lambda^{2}+q[n] \lambda\right) \Psi^{[n]}=U^{[n]} \Psi^{[n]} \\
\Psi_{t}^{[n]}=\left(2 i \sigma \lambda^{4}+V_{3}^{[n]} \lambda^{3}+V_{2}^{[n]} \lambda^{2}+V_{1}^{[n]} \lambda\right) \Psi^{[n]}=V^{[n]} \Psi^{[n]},
\end{gathered}
$$

where

$$
\begin{gathered}
q[n]=\left(\begin{array}{cc}
0 & q[n] \\
-q[n](-x,-t) & 0
\end{array}\right), \\
V_{3}^{[n]}=2 q[n], \quad V_{2}^{[n]}=i Q^{[n] 2}, \\
V_{1}^{[n]}=Q^{[n] 3}+i q[n]_{x} \sigma=\left(\begin{array}{c}
0 \\
i q[n]_{x}(-x,-t)+q^{[n] 2}(-x,-t) q[n]
\end{array} \begin{array}{c}
-i q[n]_{x}-q^{[n] 2} q[n](-x,-t) \\
0
\end{array}\right) .
\end{gathered}
$$

Using the same derivation method as the one-fold DT formula, yields

$$
q[n]=\frac{a_{n}}{d_{n}} q-2 i \frac{b_{n-1}}{d_{n}}, \quad q[n](-x,-t)=\frac{d_{n}}{a_{n}} q(-x,-t)-2 i \frac{c_{n-1}}{a_{n}} .
$$

Furthermore, the determinants of $a_{n}, d_{n}, b_{n-1}$ and $c_{n-1}$ can be given by using the kernel problem of Eq.(21) and Eq.22) respectively. And then substitute $a_{n}, d_{n}, b_{n-1}$ and $c_{n-1}$ into Eq.(23), the concrete expression of the new solutions $(q[n], q[n](-x,-t))$ can be seen in the theorem as below.

Theorem 3 Let $\Psi_{j}=\left(\begin{array}{c}\phi_{j} \\ \varphi_{j}\end{array}\right), j=1,2, \ldots n$ be distinct solutions related to $\lambda_{j}$ of the spectral problem (3) and (4), then the new solutions $(q[n], q[n](-x,-t))$ given by the following DT formula of the Eq.221.

$$
q[n]=\frac{W_{11}^{2}}{W_{21}^{2}} q+2 i \frac{W_{11} W_{12}}{W_{21}^{2}}, \quad q[n](-x,-t)=\frac{W_{21}^{2}}{W_{11}^{2}} q(-x,-t)+2 i \frac{W_{21} W_{22}}{W_{11}^{2}},
$$

where $W_{11}, W_{12}$, and $W_{21}$ have different forms depending on the parity of $N$. When $n=2 k$,

$$
W_{11}=\left|\begin{array}{cccccc}
\lambda_{1}^{n-1} \varphi_{1} & \lambda_{1}^{n-2} \phi_{1} & \lambda_{1}^{n-3} \varphi_{1} & \ldots & \lambda_{1} \varphi_{1} & \phi_{1} \\
\lambda_{2}^{n-1} \varphi_{2} & \lambda_{2}^{n-2} \phi_{2} & \lambda_{2}^{n-3} \varphi_{2} & \ldots & \lambda_{2} \varphi_{2} & \phi_{2} \\
\vdots & \vdots & \vdots & \vdots & \vdots & \vdots \\
\lambda_{n}^{n-1} \varphi_{n} & \lambda_{n}^{n-2} \phi_{n} & \lambda_{n}^{n-3} \varphi_{n} & \ldots & \lambda_{n} \varphi_{n} & \phi_{n}
\end{array}\right|
$$




$$
\begin{aligned}
& W_{12}=\left|\begin{array}{cccccc}
\lambda_{1}^{n} \phi_{1} & \lambda_{1}^{n-2} \phi_{1} & \lambda_{1}^{n-3} \varphi_{1} & \ldots & \lambda_{1} \varphi_{1} & \phi_{1} \\
\lambda_{2}^{n} \phi_{2} & \lambda_{2}^{n-2} \phi_{2} & \lambda_{2}^{n-3} \varphi_{2} & \ldots & \lambda_{2} \varphi_{2} & \phi_{2} \\
\vdots & \vdots & \vdots & \vdots & \vdots & \vdots \\
\lambda_{n}^{n} \phi_{n} & \lambda_{n}^{n-2} \phi_{n} & \lambda_{n}^{n-3} \varphi_{n} & \ldots & \lambda_{n} \varphi_{n} & \phi_{n}
\end{array}\right| \\
& W_{21}=\left|\begin{array}{cccccc}
\lambda_{1}^{n-1} \phi_{1} & \lambda_{1}^{n-2} \varphi_{1} & \lambda_{1}^{n-3} \phi_{1} & \ldots & \lambda_{1} \phi_{1} & \varphi_{1} \\
\lambda_{2}^{n-1} \phi_{2} & \lambda_{2}^{n-2} \varphi_{2} & \lambda_{2}^{n-3} \phi_{2} & \ldots & \lambda_{2} \phi_{2} & \varphi_{2} \\
\vdots & \vdots & \vdots & \vdots & \vdots & \vdots \\
\lambda_{n}^{n-1} \phi_{n} & \lambda_{n}^{n-2} \varphi_{n} & \lambda_{n}^{n-3} \phi_{n} & \ldots & \lambda_{n} \phi_{n} & \varphi_{n}
\end{array}\right|, \\
& W_{22}=\left|\begin{array}{cccccc}
\lambda_{1}^{n} \varphi_{1} & \lambda_{1}^{n-2} \varphi_{1} & \lambda_{1}^{n-3} \phi_{1} & \ldots & \lambda_{1} \phi_{1} & \varphi_{1} \\
\lambda_{1}^{n} \varphi_{1} & \lambda_{2}^{n-2} \varphi_{2} & \lambda_{2}^{n-3} \phi_{2} & \ldots & \lambda_{2} \phi_{2} & \varphi_{2} \\
\vdots & \vdots & \vdots & \vdots & \vdots & \vdots \\
\lambda_{n}^{n} \varphi_{n} & \lambda_{n}^{n-2} \varphi_{n} & \lambda_{n}^{n-3} \phi_{n} & \ldots & \lambda_{n} \phi_{n} & \varphi_{n}
\end{array}\right| .
\end{aligned}
$$

When $n=2 k+1$,

$$
\begin{gathered}
W_{11}=\left|\begin{array}{cccccc}
\lambda_{1}^{n-1} \varphi_{1} & \lambda_{1}^{n-2} \phi_{1} & \lambda_{1}^{n-3} \varphi_{1} & \ldots & \lambda_{1} \phi_{1} & \varphi_{1} \\
\lambda_{2}^{n-1} \varphi_{2} & \lambda_{2}^{n-2} \phi_{2} & \lambda_{2}^{n-3} \varphi_{2} & \ldots & \lambda_{2} \phi_{2} & \varphi_{2} \\
\vdots & \vdots & \vdots & \vdots & \vdots & \vdots \\
\lambda_{n}^{n-1} \varphi_{n} & \lambda_{n}^{n-2} \phi_{n} & \lambda_{n}^{n-3} \varphi_{n} & \ldots & \lambda_{n} \phi_{n} & \varphi_{n}
\end{array}\right|, \\
W_{12}=\left|\begin{array}{cccccc}
\lambda_{1}^{n} \phi_{1} & \lambda_{1}^{n-2} \phi_{1} & \lambda_{1}^{n-3} \varphi_{1} & \ldots & \lambda_{1} \phi_{1} & \varphi_{1} \\
\lambda_{2}^{n} \phi_{2} & \lambda_{2}^{n-2} \phi_{2} & \lambda_{2}^{n-3} \varphi_{2} & \ldots & \lambda_{2} \phi_{2} & \varphi_{2} \\
\vdots & \vdots & \vdots & \vdots & \vdots & \vdots \\
\lambda_{n}^{n} \phi_{n} & \lambda_{n}^{n-2} \phi_{n} & \lambda_{n}^{n-3} \varphi_{n} & \ldots & \lambda_{n} \phi_{n} & \varphi_{n}
\end{array}\right|, \\
W_{21}=\left|\begin{array}{cccccc}
\lambda_{1}^{n-1} \phi_{1} & \lambda_{1}^{n-2} \varphi_{1} & \lambda_{1}^{n-3} \phi_{1} & \ldots & \lambda_{1} \varphi_{1} & \phi_{1} \\
\lambda_{2}^{n-1} \phi_{2} & \lambda_{2}^{n-2} \varphi_{2} & \lambda_{2}^{n-3} \phi_{2} & \ldots & \lambda_{2} \varphi_{2} & \phi_{2} \\
\vdots & \vdots & \vdots & \vdots & \vdots & \vdots \\
\lambda_{n}^{n-1} \phi_{n} & \lambda_{n}^{n-2} \varphi_{n} & \lambda_{n}^{n-3} \phi_{n} & \ldots & \lambda_{n} \varphi_{n} & \phi_{n}
\end{array}\right|, \\
W_{22}= \\
\begin{array}{cccccc}
\lambda_{1}^{n} \varphi_{1} & \lambda_{1}^{n-2} \varphi_{1} & \lambda_{1}^{n-3} \phi_{1} & \ldots & \lambda_{1} \varphi_{1} & \phi_{1} \\
\lambda_{1}^{n} \varphi_{1} & \lambda_{2}^{n-2} \varphi_{2} & \lambda_{2}^{n-3} \phi_{2} & \ldots & \lambda_{2} \varphi_{2} & \phi_{2} \\
\vdots & \vdots & \vdots & \vdots & \vdots & \vdots \\
\lambda_{n}^{n} \varphi_{n} & \lambda_{n}^{n-2} \varphi_{n} & \lambda_{n}^{n-3} \phi_{n} & \ldots & \lambda_{n} \varphi_{n} & \phi_{n}
\end{array} \mid .
\end{gathered}
$$

\section{Exact solutions of reverse-space-time DNLS equation}

\subsection{Symmetric relation of the eigenfunctions}

In order to obtain rich nontrivial solutions, in this section, the symmetric relation of the eigenfunction corresponding to the spectrum problem Eq.(3) and Eq. (4) will be given in the form of lemma.

Lemma 1 The eigenfunctions admit the following symmetry condition:

$$
\left(\begin{array}{c}
\phi\left(x, t ; \lambda_{j}\right) \\
\varphi\left(x, t ; \lambda_{j}\right)
\end{array}\right)=\left(\begin{array}{c}
\varphi\left(-x,-t ; \lambda_{j}\right) \\
\phi\left(-x,-t ; \lambda_{j}\right)
\end{array}\right)
$$


where $\left(\begin{array}{c}\phi\left(x, t ; \lambda_{j}\right) \\ \varphi\left(x, t ; \lambda_{j}\right)\end{array}\right)$ is an eigenfunction associated with $\lambda=\lambda_{j}$.

Proof Form the $x$ part of the Lax pair Eq.(3) and Eq.(4), one has

$$
\left(\begin{array}{c}
\phi\left(x, t ; \lambda_{j}\right) \\
\varphi\left(x, t ; \lambda_{j}\right)
\end{array}\right)_{x}=\left(\begin{array}{cc}
i \lambda_{j}^{2} & q(x, t) \lambda_{j} \\
-q(-x,-t) \lambda_{j} & -i \lambda_{j}^{2}
\end{array}\right)\left(\begin{array}{c}
\phi\left(x, t ; \lambda_{j}\right) \\
\varphi\left(x, t ; \lambda_{j}\right)
\end{array}\right) .
$$

Let $x=-x, t=-t$ then

$$
\left(\begin{array}{c}
\phi\left(-x,-t ; \lambda_{j}\right) \\
\varphi\left(-x,-t ; \lambda_{j}\right)
\end{array}\right)_{x}=\left(\begin{array}{cc}
-i \lambda_{j}^{2} & -q(-x,-t) \lambda_{j} \\
q(x, t) \lambda_{j} & i \lambda_{j}^{2}
\end{array}\right)\left(\begin{array}{c}
\phi\left(-x,-t ; \lambda_{j}\right) \\
\varphi\left(-x,-t ; \lambda_{j}\right)
\end{array}\right)
$$

or

$$
\left(\begin{array}{l}
\varphi\left(-x,-t ; \lambda_{j}\right) \\
\phi\left(-x,-t ; \lambda_{j}\right)
\end{array}\right)_{x}=\left(\begin{array}{cc}
i \lambda_{j}^{2} & q(x, t) \lambda_{j} \\
-q(-x,-t) \lambda_{j} & -i \lambda_{j}^{2}
\end{array}\right)\left(\begin{array}{l}
\varphi\left(-x,-t ; \lambda_{j}\right) \\
\phi\left(-x,-t ; \lambda_{j}\right)
\end{array}\right)
$$

So

$$
\left(\begin{array}{c}
\varphi\left(-x,-t ; \lambda_{j}\right) \\
\phi\left(-x,-t ; \lambda_{j}\right)
\end{array}\right) \text { and }\left(\begin{array}{c}
\phi\left(-x,-t ; \lambda_{j}\right) \\
\varphi\left(-x,-t ; \lambda_{j}\right)
\end{array}\right)
$$

satisfy the same spectral problem. Taking a similar procedure, the symmetry property also holds for the $t$ part of the Lax pair. That means, if $\left(\begin{array}{c}\phi\left(x, t ; \lambda_{j}\right) \\ \varphi\left(x, t ; \lambda_{j}\right)\end{array}\right)$ is the eigenfunction of Eq. (3) and Eq.(4) corresponding to $\lambda_{j}$, then so is $\left(\begin{array}{c}\varphi\left(-x,-t ; \lambda_{j}\right) \\ \phi\left(-x,-t ; \lambda_{j}\right)\end{array}\right)$.

\subsection{Exact solutions generated from zero seed solution}

Let $q=q(-x,-t)=0$ in Eq.(3) and Eq. (4), then the eigenfunction can be solved as

$$
\Psi_{k}=\left(\begin{array}{c}
\phi_{k} \\
\varphi_{k}
\end{array}\right)=\left(\begin{array}{c}
e^{i\left(\lambda_{k}^{2} x+2 \lambda_{k}^{4} t\right)} \\
e^{-i\left(\lambda_{k}^{2} x+2 \lambda_{k}^{4} t\right)}
\end{array}\right)
$$

Exact solutions of reverse-space-time DNLS equation can be obtained by substituting the eigenfunction Eq. 25] into the DT formula Eq. 24].

For $\mathrm{N}=1$ : the solution with $\lambda_{1}=\alpha_{1}+i \beta_{1}$ can be expressed as follows

$$
\begin{aligned}
q[1] & =\left(2 i \alpha_{1}-2 \beta_{1}\right) e^{i A+B}, \\
A & =-2\left(2 \alpha_{1}^{4} t-12 \alpha_{1}^{2} \beta_{1}^{2} t-2 \beta_{1}^{4} t+\alpha_{1}^{2} x-\beta_{1}^{2} x\right), \\
B & =2\left(8 \alpha_{1}^{3} \beta_{1} t-8 \alpha_{1} \beta_{1}^{3} t+2 \alpha_{1} \beta_{1} x\right) .
\end{aligned}
$$

When $\alpha_{1} \beta_{1}=0,|q[1]|^{2}=4\left(\alpha_{1}^{2}+\beta_{1}^{2}\right)$, which is a plane wave with heigh of $2 \sqrt{\alpha_{1}^{2}+\beta_{1}^{2}}$.

When $\alpha_{1} \beta_{1} \neq 0,|q[1]|^{2}=4\left(\alpha_{1}^{2}+\beta_{1}^{2}\right) e^{8 \alpha_{1} \beta_{1}\left(4 \alpha_{1}^{2} t-4 \beta_{1}^{2} t+x\right)}$, which is an exponentially growing curved surface wave.

For $\mathrm{N}=2$ : Let $\lambda_{1}=\alpha_{1}+i \beta_{1}, \lambda_{2}=\alpha_{2}+i \beta_{2}$, an explicit expression for $q[2]$ as follows

$$
q[2]=\frac{2 i \zeta_{1} e^{i A_{1}} B\left[\zeta_{1} B \sin A_{4} \sin A_{3}+\zeta_{2} \sin \left(A_{3}-A_{4}\right)+\zeta_{1} \cos A_{4} \cos A_{3}\right]}{\left(\zeta_{2} \sin \left(A_{3}-A_{4}\right)-\zeta_{1} \cos \left(A_{3}+A_{4}\right)\right)^{2}},
$$


where

$$
\begin{aligned}
& B=\cosh A_{2}-\sinh A_{2}, \\
& A_{1}=\left(2 \alpha_{1}^{4}-12 \alpha_{1}^{2} \beta_{1}^{2}+2 \alpha_{2}^{4}-12 \alpha_{2}^{2} \beta_{2}^{2}+2 \beta_{1}^{4}+2 \beta_{2}^{4}\right) t-\left(-\alpha_{1}^{2}-\alpha_{2}^{2}+\beta_{1}^{2}+\beta_{2}^{2}\right) x, \\
& A_{2}=\left(8 \alpha_{1}^{3} \beta_{1}-8 \alpha_{1} \beta_{1}^{3}+8 \alpha_{2}^{3} \beta_{2}-8 \alpha_{2} \beta_{2}^{3}\right) t+2 x\left(\alpha_{1} \beta_{1}+\alpha_{2} \beta_{2}\right), \\
& A_{3}=2 \alpha_{2}^{4} t+8 i \alpha_{2}^{3} \beta_{2} t+\left(-12 \beta_{2}^{2} t+x\right) \alpha_{2}^{2}-8 i\left(\beta_{2}^{2} t-\frac{1}{4} x\right) \beta_{2} \alpha_{2}+2 \beta_{2}^{4} t-\beta_{2}^{2} x, \\
& A_{4}=2 \alpha_{1}^{4} t+8 i \alpha_{1}^{3} \beta_{1} t+\left(-12 \beta_{1}^{2} t+x\right) \alpha_{1}^{2}-8 i\left(\beta_{1}^{2} t-\frac{1}{4} x\right) \beta_{1} \alpha_{1}+2 \beta_{1}^{4} t-\beta_{1}^{2} x, \\
& \zeta_{1}=\alpha_{1}+i \beta_{1}-\alpha_{2}-i \beta_{2}, \quad \zeta_{2}=i \alpha_{1}+i \alpha_{2}-\beta_{1}-\beta_{2} .
\end{aligned}
$$

The rational solution and periodic solutions can be constructed as follows by lemma 1 and formula (24).

1. $\lambda_{2}= \pm \lambda_{1}^{*}$, then $A_{2}=0, A_{3}+A_{4}=0$ in the Eq.(27). we find that the solution $q[2]$ representation as the bell-shaped soliton solution. Without loss of generality, let $\lambda_{2}=-\lambda_{1}^{*}$, then

$$
q[2]=-2 \zeta_{1} e^{i H} \frac{\zeta_{1} \cosh (F-i H)+i \zeta_{2} \sinh (F-i H)}{\left(i \zeta_{2} \sinh (F-i H)-m\right)^{2}},
$$

where

$$
\begin{aligned}
& \left.H=2\left(\alpha_{1}^{2}-\beta_{1}^{2}\right) x+4\left(\beta_{1}^{4}-6 \alpha_{1}^{2} \beta_{1}^{2}+\alpha_{1}^{4}\right) t\right), \\
& F=16 \alpha_{1}^{3} \beta_{1} t-16 \alpha_{1} \beta_{1}^{3} t+4 \alpha_{1} \beta_{1} x .
\end{aligned}
$$

Furthermore, $q[2]$ can become a rational solution by the limit technique $\alpha_{1} \rightarrow 0$,

$$
q[2]_{r}=\frac{e^{2 i \beta_{1}^{2}\left(2 \beta_{1}^{2} t-x\right)}\left(64 i t \beta_{1}^{5}-16 i x \beta_{1}^{3}-4 \beta_{1}\right)}{1-256 \beta_{1}^{8} t^{2}+128 \beta_{1}^{6} t x+\left(32 i t-16 x^{2}\right) \beta_{1}^{4}-8 i \beta_{1}^{2} x},
$$

$\beta_{1}$ is an arbitrary real constant.

$$
\left|q[2]_{r}\right|^{2}=\frac{16 \beta_{1}^{2}}{\left(16 \beta_{1}^{4} t-4 \beta_{1}^{2} x\right)^{2}+1} .
$$

Obviously, $q[2]_{r}$ is an analytical solution at whole $(x, t)$ plane, and its trajectory is defined explicitly by $x=4 \beta_{1}^{2} t$ (see Fig $1(\mathrm{a})$.

2. When $\lambda_{1}, \lambda_{2}$ are pure imaginary number or real number, then $A_{2}=0$ in the Eq. 227). $q[2]$ represented by the form of periodic solutions (see Fig $1(\mathrm{~b})$. For example, $\lambda_{1}=i \beta_{1}, \lambda_{2}=i \beta_{2}$,

$$
\begin{gathered}
q[2]=2 i \zeta_{1} e^{i\left(K_{1}+K_{2}\right)} \frac{\zeta_{1} \cos \left(K_{1}-K_{2}\right)+\zeta_{2} \sin \left(K_{1}-K_{2}\right)}{\left[-\zeta_{1} \cos \left(K_{1}+K_{2}\right)+\zeta_{2} \sin \left(K_{1}-K_{2}\right)\right]^{2}}, \\
K_{1}=2 \beta_{2}^{2}\left(2 \beta_{2}^{2} t-x\right), \quad K_{2}=2 \beta_{1}^{2}\left(2 \beta_{1}^{2} t-x\right) .
\end{gathered}
$$




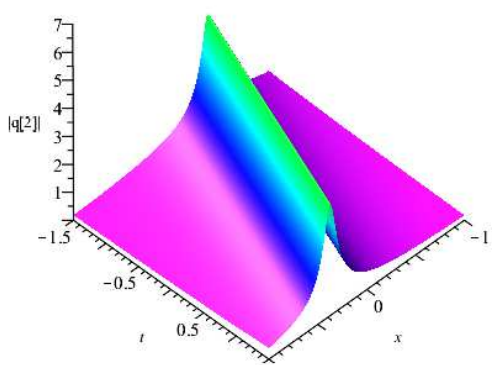

(a) Rational solution

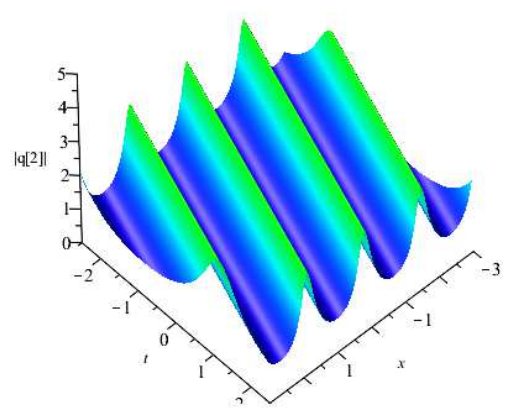

(b) Periodic solution

Fig. 1: Rational solution and periodic solution generated from zero seed solution: (a) $\beta_{1}=\frac{1}{2}$; (b) $\beta_{1}=1, \beta_{2}=-1$.

\subsection{Exact solutions generated from non-zero seed solution}

Starting from the seed solutions $q(x, t)=c e^{i(a x+b t)}, q(-x,-t)=c e^{-i(a x+b t)}, b=-a c^{2}+a^{2}$. And $c$ denoting the height of the background. Taking the following transformation

$$
\Psi(x, t ; \lambda)=e^{\frac{i}{2}(a x+b t) \sigma} \Phi(x, t ; \lambda),
$$

then

$$
\begin{gathered}
\Phi_{x}(x, t ; \lambda)=\widehat{M} \Phi(x, t ; \lambda)=\left(\begin{array}{cc}
-\frac{\mathrm{i}}{2}\left(\mathrm{a}-2 \lambda^{2}\right) & c \lambda \\
-c \lambda & \frac{\mathrm{i}}{2}\left(\mathrm{a}-2 \lambda^{2}\right)
\end{array}\right) \Phi(x, t ; \lambda), \\
\Phi_{t}(x, t ; \lambda)=\widehat{N} \Phi(x, t ; \lambda)=\left(\begin{array}{cc}
-\frac{\mathrm{i}}{2}\left(a-c^{2}+2 \lambda^{2}\right)\left(a-2 \lambda^{2}\right) & c \lambda\left(a-c^{2}+2 \lambda^{2}\right) \\
-c \lambda\left(a-c^{2}+2 \lambda^{2}\right) & \frac{\mathrm{i}}{2}\left(a-c^{2}+2 \lambda^{2}\right)\left(a-2 \lambda^{2}\right)
\end{array}\right) \Phi(x, t ; \lambda) .
\end{gathered}
$$

Thus, solving the Lax pair Eq.(3) and Eq.(4) is equivalent to solving equations Eq.(33) and Eq.(34). Solving the equations Eq.(33) and Eq.(34), it leads to

$$
\begin{gathered}
\Phi_{1 k}=\left(\begin{array}{c}
\phi_{11 k} \\
\phi_{12 k}
\end{array}\right)=\left(\begin{array}{l}
\mathrm{e}^{\frac{1}{2} t R\left(a-c^{2}+2 \lambda_{k}^{2}\right)+\frac{1}{2} x R} \\
\frac{i a-2 i \lambda_{k}^{2}+R}{2 c \lambda_{k}} \mathrm{e}^{\frac{1}{2} t R\left(a-c^{2}+2 \lambda_{k}^{2}\right)+\frac{1}{2} x R}
\end{array}\right), \\
\Phi_{2 k}=\left(\begin{array}{c}
\phi_{21 k} \\
\phi_{22 k}
\end{array}\right)=\left(\begin{array}{l}
\mathrm{e}^{-\frac{1}{2} t R\left(-c^{2}+2 \lambda_{k}^{2}+a\right)-\frac{1}{2} x R \lambda_{k}} \\
\frac{i a-2 i \lambda_{k}^{2}-R}{2 c \lambda_{k}} \mathrm{e}^{-\frac{1}{2} t R\left(-c^{2}+2 \lambda_{k}^{2}+a\right)-\frac{1}{2} x R}
\end{array}\right), \\
R=\sqrt{-4 c^{2} \lambda_{k}^{2}-4 \lambda_{k}^{4}+4 a \lambda_{k}^{2}-a^{2}} .
\end{gathered}
$$

Due to Eq.(5), we can get

$$
\begin{gathered}
\Psi_{1 k}=\left(\begin{array}{l}
\psi_{11 k} \\
\psi_{12 k}
\end{array}\right)=\left(\begin{array}{l}
\mathrm{e}^{\frac{1}{2} t R\left(-c^{2}+2 \lambda_{k}^{2}+a\right)+\frac{1}{2} x R+\frac{1}{2}(i(a x+b t))} \\
\frac{i a-2 i \lambda_{k}^{2}+R}{2 c \lambda_{k}} \mathrm{e}^{\frac{1}{2} t R\left(-c^{2}+2 \lambda_{k}^{2}+a\right)+\frac{1}{2} x R-\frac{1}{2}(i(a x+b t))}
\end{array}\right), \\
\Psi_{2 k}=\left(\begin{array}{l}
\psi_{21 k} \\
\psi_{22 k}
\end{array}\right)=\left(\begin{array}{l}
\mathrm{e}^{-\frac{1}{2} t R\left(-c^{2}+2 \lambda_{k}^{2}+a\right)-\frac{1}{2} x R \lambda_{k}+\frac{1}{2}(i(a x+b t))} \\
\frac{i a-2 i \lambda_{k}^{2}-R}{2 c \lambda_{k}} \mathrm{e}^{-\frac{1}{2} t R\left(-c^{2}+2 \lambda_{k}^{2}+a\right)-\frac{1}{2} x R-\frac{1}{2}(i(a x+b t))}
\end{array}\right) .
\end{gathered}
$$


Substitute different solutions $\Psi_{1 k}, \Psi_{2 k}$ of the spectral problem Eq.(3) and Eq.(4) into Eq.(24), can only get trivial solutions of Eq. (2). In order to get richer solutions, the new eigenfunctions associated with $\lambda_{k}$ can be expressed by the principle of the superposition of the linear differential equation.

$$
\begin{aligned}
& \phi_{k}=\psi_{11 k}+\psi_{21 k}+\psi_{12 k}(-x,-t)+\psi_{22 k}(-x,-t), \\
& \psi_{k}=\psi_{12 k}+\psi_{22 k}+\psi_{11 k}(-x,-t)+\psi_{21 k}(-x,-t) .
\end{aligned}
$$

For $N=1$ : Substitute eigenfunction Eq. 35 into (24) we can get more complex but very meaningful new solutions. For simple, let $\lambda_{1}=i \beta_{1}$, then

$$
|q[1]|^{2}=\frac{\left[\left(i R_{1}-\omega_{1}\right) e^{\omega_{2}+\omega_{5}}-\left(i R_{1}+\omega_{1}\right) e^{\omega_{5}}\right]\left[\left(\omega_{3}+\omega_{4}\right) e^{\omega_{2}}+\omega_{3}-\omega_{4}\right]}{\left[\left(i R_{1}+\omega_{1}\right) e^{\omega_{2}}-i R_{1}+\omega_{1}\right]^{2}},
$$

where

$$
\begin{aligned}
& \omega_{1}=2 \beta_{1}^{2}+2 \beta_{1} c+a, \\
& \omega_{2}=R_{1}\left[\left(-2 \beta_{1}^{2}-c^{2}+a\right) t+x\right], \\
& \omega_{3}=4 \beta_{1}^{3}+2 \beta_{1}^{2} c+\left(2 a-2 c^{2}\right) \beta_{1}-a c, \\
& \omega_{4}=i R_{1}\left(2 \beta_{1}+c\right), \\
& \omega_{5}=i a c^{2} t-i a^{2} t-i a x, \\
& R_{1}=\sqrt{4 c^{2} \beta_{1}^{2}-\left(2 \beta_{1}^{2}+a\right)^{2}} .
\end{aligned}
$$

The trajectory of the solution $\sqrt{36}$ is

$$
x=\left(2 \beta_{1}^{2}+c^{2}-a\right) t
$$

and $|q[1]|^{2} \rightarrow c^{2}-2 a$ at $x \rightarrow \infty, t \rightarrow \infty$. There are four roots $-\frac{c}{2}+\frac{\sqrt{c^{2}-2 a}}{2},-\frac{c}{2}-\frac{\sqrt{c^{2}-2 a}}{2}$, $\frac{c}{2}+\frac{\sqrt{c^{2}-2 a}}{2}$ and $\frac{c}{2}-\frac{\sqrt{c^{2}-2 a}}{2}$ via solve $4 c^{2} \beta_{1}^{2}-\left(2 \beta_{1}^{2}+a\right)^{2}=0$.

1. When $c^{2}>2 a$, Eq. 36 can generate soliton solutions. More profound, we find that Eq. 36. can generate a dark soliton when $c^{2}>2 a>0, \frac{c}{2}+\frac{\sqrt{c^{2}-2 a}}{2}>\beta_{1}>\frac{c}{2}-\frac{\sqrt{c^{2}-2 a}}{2}$ or $c^{2}>0>2 a$, $\frac{c}{2}+\frac{\sqrt{c^{2}-2 a}}{2}>\beta_{1}>-\frac{c}{2}+\frac{\sqrt{c^{2}-2 a}}{2}$ (see Fig 2(a). Eq. (36) can generate a bright soliton if $c^{2}>2 a>0,-\frac{c}{2}+\frac{\sqrt{c^{2}-2 a}}{2}>\beta_{1}>-\frac{c}{2}-\frac{\sqrt{c^{2}-2 a}}{2}$ or $c^{2}>0>2 a, \frac{c}{2}-\frac{\sqrt{c^{2}-2 a}}{2}>\beta_{1}>-\frac{c}{2}-\frac{\sqrt{c^{2}-2 a}}{2}$ (see Fig 2(b)]. Eq. $(36)$ can generate periodic solutions when $\beta_{1}$ belongs to other intervals.

2. $c^{2} \leq 2 a, \forall \beta_{1} \in R$, Eq.(36) also generate periodic solution (see Fig 2(c)].

For $\mathrm{N}=2$ : The two-fold DT formula 24) of the reverse-space-time DNLS equation implies a solution

$$
q[2]=\frac{-2\left[i\left(\lambda_{1}^{2}-\lambda_{2}^{2}\right) \phi_{1} \phi_{2}-\frac{1}{2} \lambda_{2} \phi_{1} \psi_{2}+\frac{1}{2} \lambda_{1} q \phi_{2} \psi_{1}\right]\left(-\lambda_{1} \phi_{2} \psi_{1}+\lambda_{2} \phi_{1} \psi_{2}\right)}{\left(\lambda_{1} \phi_{1} \psi_{2}-\lambda_{2} \phi_{2} \psi_{1}\right)^{2}}
$$

where

$$
\left(\begin{array}{l}
\phi_{2} \\
\psi_{2}
\end{array}\right)=\left(\begin{array}{c}
\psi_{1}\left(-x,-t ; \lambda_{2}\right) \\
\phi_{1}\left(-x,-t ; \lambda_{2}\right)
\end{array}\right)
$$

Taking $\lambda_{1}=\alpha_{1}+i \beta_{1}, \lambda_{2}=\alpha_{2}+i \beta_{2}$ in Eq.39). Different nonsingular solutions of Eq. 22 can be found according to different reduced methods of spectrum parameter $\lambda_{k}$. 


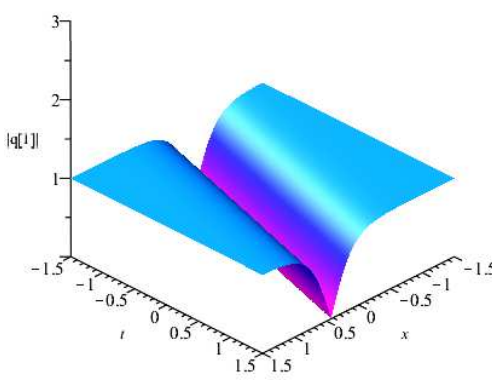

(a) Dark soliton

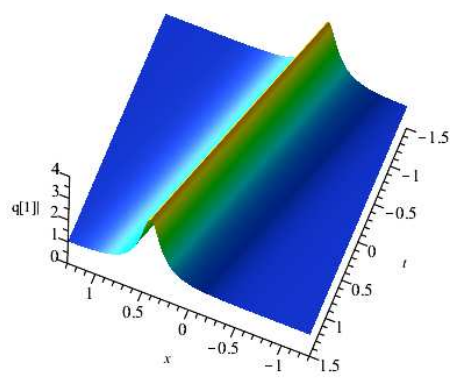

(b) Bright soliton

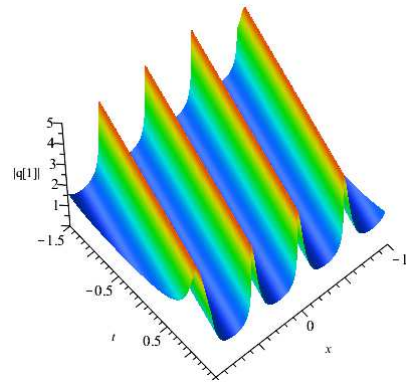

(c) Periodic solution

Fig. 2: Dark soliton, bright soliton and periodic solution generated from non-zero seed solution: (a) $a=1, c=\sqrt{3}, \beta_{1}=-\frac{\sqrt{3}}{2}$; (b) $a=1, c=\sqrt{3}, \beta_{1}=\frac{\sqrt{3}}{2}$; (c) $a=1, c=\sqrt{3}, \beta_{1}=\frac{3}{2}$.

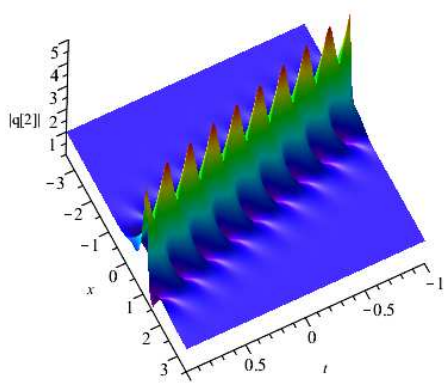

(a) General breathers

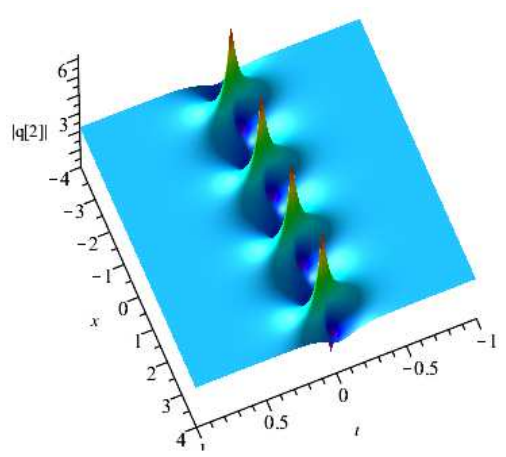

(c) Akmediev breathers

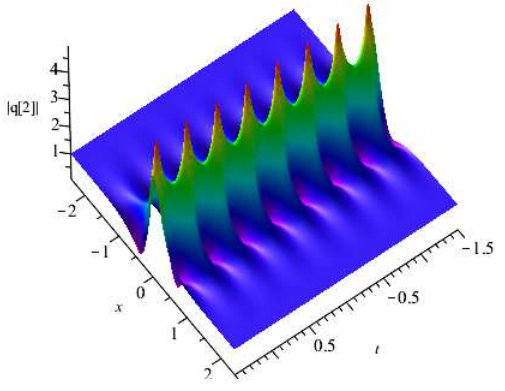

(b) Ma breathers

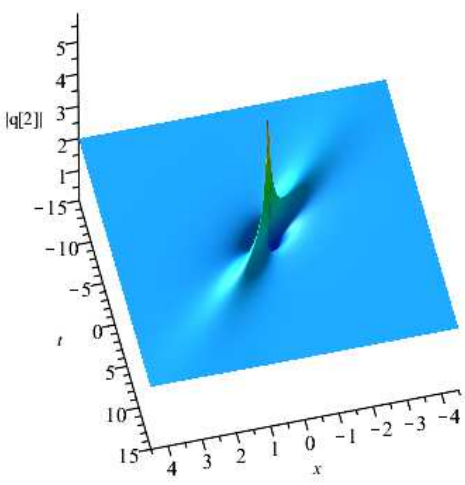

(d) Rogue wave

Fig. 3: Breathers and rogue waves generated from non-zero seed solution: (a) $a=4, c=1$, $\lambda_{1}=1+i, \lambda_{2}=-1+i$; (b) $a=1, c=1, \lambda_{1}=1+i, \lambda_{2}=-1+i$; (c) $a=5, c=\sqrt{5}, \lambda_{1}=1+i$, $\lambda_{2}=-1+i ;(\mathrm{d}) \alpha_{1}=\frac{1}{2}, \beta_{1}=1$; 
1. $\lambda_{2}= \pm \lambda_{1}^{*}$, now $q[2]$ is breather solution. For example, take $\lambda_{1}=\alpha_{1}+i \beta_{1}, \lambda_{2}=-\alpha_{1}+i \beta_{1}$, in general, the solution evolves periodically along the straight line with a certain angle of $x$ axis and $t$ axis (see Fig $3(\mathrm{a})$. Specifically, when $a=2 \alpha_{1}^{2}-2 \beta_{1}^{2}+c^{2}$, i.e. $\operatorname{Im}\left(-a^{2}-4 \lambda_{1}^{4}-4 \lambda_{1}^{2}\left(c^{2}-a\right)\right)=$ 0 , then the classical Ma breathers (time periodic breather) can be seen in Fig. $3(\mathrm{~b})$. And the Akhmediev breathers (space periodic breather) can be seen in Fig $3(\mathrm{c})$. By letting $x \rightarrow \infty, t \rightarrow$ $\infty$, so $|q[2]|^{2} \rightarrow c^{2}$, the trajectory of this solution is $x=-4 \alpha_{1}^{2} t+4 \beta_{1}^{2} t$.

Only one localized peak remains from the periodic train when the period of the breather tends to the infinity, then the rogue wave comes into being. Next, the rogue wave $q_{r}$ of Eq. (2) can be derived by $c \rightarrow-2 \beta_{1}$.

$$
\left|q_{r}\right|^{2}=\frac{m_{1} t^{4}+m_{2} t^{3}+m_{3} t^{2}+m_{4} t+m_{5}}{m_{6} t^{4}+m_{7} t^{3}+m_{8} t^{2}+m_{9} t+m_{10}},
$$

$$
\begin{aligned}
& m_{1}=262144\left(\alpha_{1}^{12} \beta_{1}^{6}+2 \alpha_{1}^{6} \beta_{1}^{12}+\beta_{1}^{18}\right), \\
& m_{2}=262144\left(\alpha_{1}^{10} \beta_{1}^{6}-\alpha_{1}^{6} \beta_{1}^{10}+\alpha_{1}^{4} \beta_{1}^{12}-\beta_{1}^{16}\right) x, \\
& m_{3}=\left(32768\left(3 \alpha_{1}^{8} \beta_{1}^{6}+\alpha_{1}^{6} \beta_{1}^{8}-4 \alpha_{1}^{4} \beta_{1}^{10}+\alpha_{1}^{2} \beta_{1}^{12}+3 \beta_{1}^{14}\right) x^{2}-6144\left(\alpha_{1}^{6} \beta_{1}^{4}-6 \alpha_{1}^{4} \beta_{1}^{6}+\beta_{1}^{10}\right)\right), \\
& m_{4}=\left(16384\left(\alpha_{1}^{6} \beta_{1}^{6}+\alpha_{1}^{4} \beta_{1}^{8}-\alpha_{1}^{2} \beta_{1}^{10}-\beta_{1}^{12}\right) x^{3}+3072\left(2 \alpha_{1}^{2} \beta_{1}^{6}+\beta_{1}^{8}-\alpha_{1}^{4} \beta_{1}^{4}\right) x\right), \\
& m_{5}=1024\left(\alpha_{1}^{4} \beta_{1}^{6}+2 \alpha_{1}^{2} \beta_{1}^{8}+\beta_{1}^{10}\right) x^{4}-128\left(3 \alpha_{1}^{2} \beta_{1}^{4}+\beta_{1}^{6}\right) x^{2}+36 \beta_{1}^{2}, \\
& m_{6}=65536\left(\alpha_{1}^{12} \beta_{1}^{4}+2 \alpha_{1}^{6} \beta_{1}^{10}+\beta_{1}^{16}\right), \\
& m_{7}=65536\left(\alpha_{1}^{10} \beta_{1}^{4}-\alpha_{1}^{6} \beta_{1}^{8}+\alpha_{1}^{4} \beta_{1}^{10}-\beta_{1}^{14}\right) x, \\
& m_{8}=8192\left(3 \alpha_{1}^{8} \beta_{1}^{4}+\alpha_{1}^{6} \beta_{1}^{6}-4 \alpha_{1}^{4} \beta_{1}^{8}+\alpha_{1}^{2} \beta_{1}^{10}+3 \beta_{1}^{12}\right) x^{2}+512\left(\alpha_{1}^{6} \beta_{1}^{2}+2 \alpha_{1}^{4} \beta_{1}^{4}-8 \alpha_{1}^{2} \beta_{1}^{6}+9 \beta_{1}^{8}\right), \\
& m_{9}=4096\left(\alpha_{1}^{6} \beta_{1}^{4}+\alpha_{1}^{4} \beta_{1}^{6}-\alpha_{1}^{2} \beta_{1}^{8}-\beta_{1}^{10}\right) x^{3}+256\left(\alpha_{1}^{4} \beta_{1}^{2}+2 \alpha_{1}^{2} \beta_{1}^{4}-5 \beta_{1}^{6}\right) x, \\
& m_{10}=256\left(\alpha_{1}^{4} \beta_{1}^{4}+2 \alpha_{1}^{2} \beta_{1}^{6}+\beta_{1}^{8}\right) x^{4}+32\left(\alpha_{1}^{2} \beta_{1}^{2}+3 \beta_{1}^{4}\right) x^{2}+1 .
\end{aligned}
$$

$\left|q_{r}\right|^{2} \rightarrow\left(2 \beta_{1}\right)^{2}$ in the above expressions 41 with $x \rightarrow \infty, t \rightarrow \infty$. After calculation and analysis, we know the maximum amplitude of $\left|q_{r}\right|^{2}$ equals to $\left(6 \beta_{1}\right)^{2}$ occurs at $x=0$ and $t=0$. This means that the maximum amplitude of the rogue wave $q_{r}$ is 3 times that of the asymptotic plane wave at infinity. The min amplitude of $\left|q_{r}\right|^{2}$ occurs at $\left(x=\sqrt{\frac{27 \alpha_{1}^{4}}{16 \beta_{1}^{2}\left(4 \alpha_{1}^{2}+\beta_{1}^{2}\right)\left(\alpha_{1}^{2}+\beta_{1}^{2}\right)^{2}}}, t=\right.$ $\sqrt{\left.\frac{3}{256 \beta_{1}^{2}\left(4 \alpha_{1}^{2}+\beta_{1}^{2}\right)\left(\alpha_{1}^{2}+\beta_{1}^{2}\right)^{2}}\right)}$ and $\left(x=-\sqrt{\frac{27 \alpha_{1}^{4}}{16 \beta_{1}^{2}\left(4 \alpha_{1}^{2}+\beta_{1}^{2}\right)\left(\alpha_{1}^{2}+\beta_{1}^{2}\right)^{2}}}, t=-\sqrt{\left.\frac{3}{256 \beta_{1}^{2}\left(4 \alpha_{1}^{2}+\beta_{1}^{2}\right)\left(\alpha_{1}^{2}+\beta_{1}^{2}\right)^{2}}\right)}\right.$, which equals to $\frac{108\left(3 \alpha_{1}^{4}-2 \alpha_{1}^{2} \beta_{1}^{2}+4 \beta_{1}^{4}\right) \alpha_{1}^{4} \beta_{1}^{2}}{169 \alpha_{1}^{8}-56 \alpha_{1}^{6} \beta_{1}^{2}+6 \alpha_{1}^{4} \beta_{1}^{4}-8 \alpha_{1}^{2} \beta_{1}^{6}+4 \beta_{1}^{8}}$. Fig. $3(\mathrm{~d})$ is plotted for the rogue wave $\left|q_{r}\right|$ with specific parameter $\alpha_{1}=\frac{1}{2}, \beta_{1}=1$. From the graph of the rogue wave $\left|q_{r}\right|$, we can see that the rogue wave $q_{r}$ has a single peak with two caves on both sides of the peak. The optical pulse $q_{r}$ only exists locally with all variables and disappears as time and space go far.

2. $\operatorname{Re}\left(\lambda_{1}\right)=\operatorname{Re}\left(\lambda_{2}\right)=0, q[2]$ is represented as a double-periodic wave solution (see Fig 4). As can be seen from the figure, there are two periodic waves with different directions in the doubleperiodic wave solution, and when the two waves with different directions are superimposed on each other, a higher wave peak can be generated. From a visual perspective, it seems that several parallel breaths are generated under the period background.

For $N=3$ : Set $\lambda_{1}=\alpha_{1}+i \beta_{1}, \lambda_{2}=-\alpha_{1}+i \beta_{1}, \lambda_{3}=i \beta_{3}$ and $a=-2 \alpha_{1}^{2}+2 \beta_{1}^{2}$. Parameter values have a great influence on the propagation direction of the breathers. For example, when $a=5, c=\sqrt{5}, \alpha_{1}=1, \beta_{1}=1$ and $\beta_{3}=\sqrt{2} i$, solution $q[3]$ is a breather on the periodic background generated by formula (24) as shown in Fig.5. We can call it Ma breathers on the periodic background due to the breather is timely periodic in Fig $5(\mathrm{a})$. However, when $a=1$, $c=1, \alpha_{1}=1, \beta_{1}=1$ and $\beta_{3}=\sqrt{2} i$ the breather is spatially periodic in Fig $5(\mathrm{~b})$ which can 

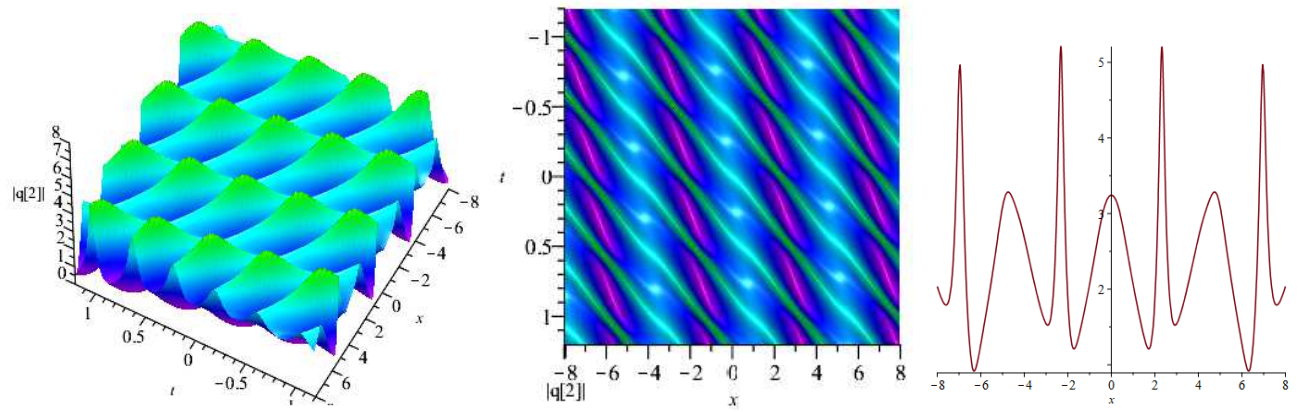

Fig. 4: double-periodic solution: $a=1, c=1, \beta_{1}=\sqrt{2}, \beta_{2}=\frac{\sqrt{2}}{2}$.

be named Akhmediev breathers on the periodic background. Similarly, when $a=1, c=1$, $\alpha_{1}=1, \beta_{1}=\frac{1}{3}$ and $\beta_{3}=\sqrt{2} i$, solution $q[3]$ is a general breather solution on periodic background (see Fig $5(\mathrm{c})$. There are some interesting new phenomenons: Under the perturbation of the periodic background, the crest of the Ma breathers is cut and the phase shift occurs at the center of the breathers.

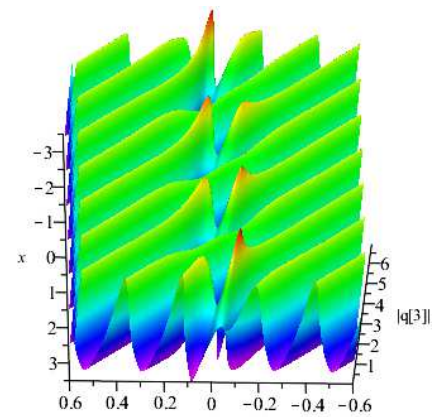

(a) Ma breathers on the periodic (b) background
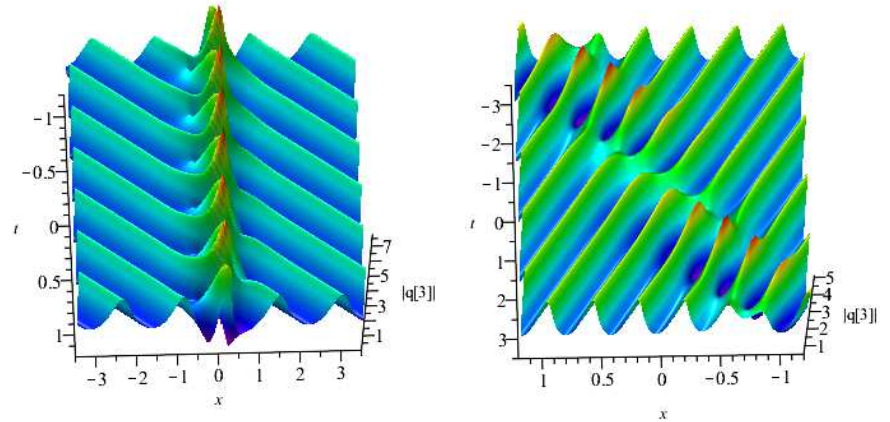

(b) Akhmediev brea
riodic background (c) General breathers on the periodic background

Fig. 5: Breathers on the periodic background: (a) $a=5, c=\sqrt{5}, \alpha_{1}=1, \beta_{1}=1, \beta_{3}=\sqrt{2} i$; (b) $a=1, c=1, \alpha_{1}=1, \beta_{1}=1, \beta_{3}=\sqrt{2} i$; (c) $a=1, c=1, \alpha_{1}=1, \beta_{1}=\frac{1}{3}, \beta_{3}=\sqrt{2} i$.

For $\mathrm{N}=4$ : Set $\lambda_{1}=\alpha_{1}+i \beta_{1}, \lambda_{2}=-\alpha_{1}+i \beta_{1}, \lambda_{3}=i \beta_{3}$ and $\lambda_{4}=i \beta_{4}$. As can be seen in Fig, 6 , the breathers on a double-periodic background generate by formula (24). The solution is a new find generated by plane wave seed solution $c e^{i\left(a x+\left(a^{2}-a c^{2}\right) t\right)}$. It is worth noting that the amplitude of breathers is greatly influenced by the periodic background. Breathers on the double-periodic background more easily observed in the $t$ direction (see Fig 6). 

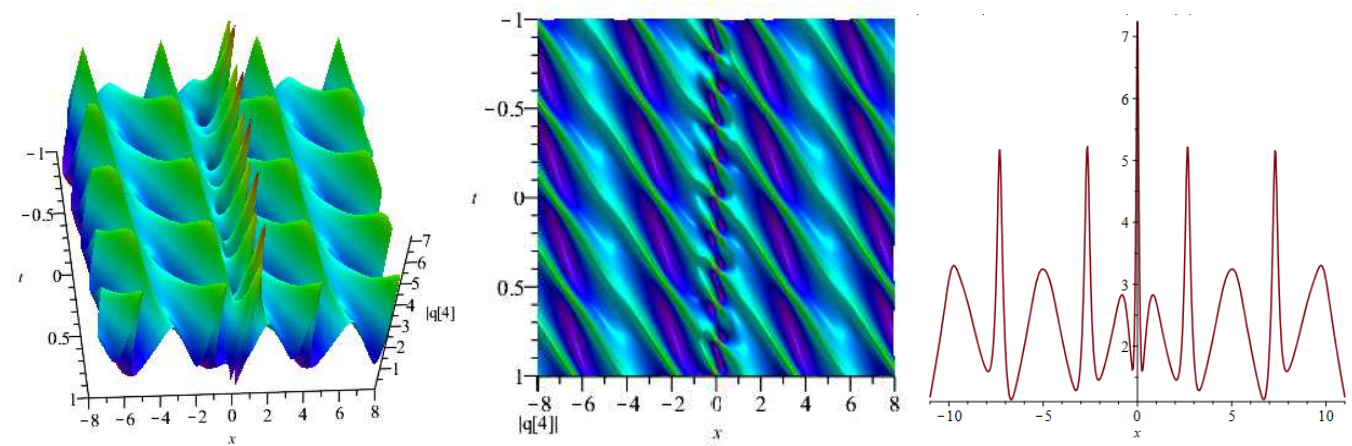

Fig. 6: Breathers on the double-periodic background generated from the non-zero seed solution: $a=1, c=1, \beta_{1}=\sqrt{2}, \beta_{2}=\frac{\sqrt{2}}{2}$.

\section{Rogue waves on the periodic and double-periodic background}

\subsection{Generalized DT for the reverse-space-time DNLS equation}

Note that the eigenfunction is degenerated when $\lambda=\frac{1}{2} \sqrt{2 a-c^{2}}-\frac{1}{2} i c$. In this case, the high-order rogue waves on the periodic and double-periodic background can be obtained.

Theorem 4 Let $\lambda_{1}=\frac{1}{2} \sqrt{2 a-c^{2}}-\frac{1}{2} i c, \lambda_{2}=-\frac{1}{2} \sqrt{2 a-c^{2}}-\frac{1}{2} i c$. When $N=2 k$, set $\lambda_{N-1}=$ $i \beta_{N-1}$ and $\lambda_{N}=i \beta_{N}$. When $N=2 k+1$, set $\lambda_{N}=i \beta_{N}$, the rogue waves on the periodic and double-periodic can be obtained as

$$
q_{n}=\frac{W_{11}^{\prime 2}}{W_{21}^{\prime 2}} q+2 i \frac{W_{11}^{\prime} W_{12}^{\prime}}{W_{21}^{\prime 2}}
$$

When $n=2 k$,

$$
\begin{aligned}
& W_{11}^{\prime}=\left|\begin{array}{cccccc}
\varphi_{[1, n-1,1]} & \phi_{[1, n-2,1]} & \varphi_{[1, n-3,1]} & \ldots & \varphi_{[1,1,1]} & \phi_{[1,0,1]} \\
\varphi_{[2, n-1,1]} & \phi_{[2, n-2,1]} & \varphi_{[2, n-3,1]} & \ldots & \varphi_{[2,1,1]} & \phi_{[2,0,1]} \\
\vdots & \vdots & \vdots & \vdots & \vdots & \vdots \\
\varphi_{[1, n-1, k-1]} & \phi_{[1, n-2, k-1]} & \varphi_{[1, n-3, k-1]} & \ldots & \varphi_{[1,1, k-1]} & \phi_{[1,0, k-1]} \\
\varphi_{[2, n-1, k-1]} & \phi_{[2, n-2, k-1]} & \varphi_{[2, n-3, k-1]} & \ldots & \varphi_{[2,1, k-1]} & \phi_{[2,0, k-1]} \\
\lambda_{n-1}^{n-1} \varphi_{n-1} & \lambda_{n-1}^{n-2} \phi_{n-1} & \lambda_{n-3}^{n-3} \varphi_{n-1} & \ldots & \lambda_{n-1} \varphi_{n-1} & \phi_{n-1} \\
\lambda_{n}^{n-1} \varphi_{n} & \lambda_{n}^{n-2} \phi_{n} & \lambda_{n}^{n-3} \varphi_{n} & \ldots & \lambda_{n} \varphi_{n} & \phi_{n}
\end{array}\right|, \\
& W_{12}^{\prime}=\left|\begin{array}{cccccc}
\phi_{[1, n, 1]} & \phi_{[1, n-2,1]} & \varphi_{[1, n-3,1]} & \ldots & \varphi_{[1,1,1]} & \phi_{[1,0,1]} \\
\phi_{[2, n, 1]} & \phi_{[2, n-2,1]} & \varphi_{[2, n-3,1]} & \ldots & \varphi_{[2,1,1]]} & \phi_{[2,0,1]} \\
\vdots & \vdots & \vdots & \vdots & \vdots & \vdots \\
\phi_{[1, n, k-1]} & \phi_{[1, n-2, k-1]} & \varphi_{[1, n-3, k-1]} & \ldots & \varphi_{[1,1, k-1]} & \phi_{[1,0, k-1]} \\
\phi_{[2, n, k-1]} & \phi_{[2, n-2, k-1]} & \varphi_{[2, n-3, k-1]} & \ldots & \varphi_{[2,1, k-1]]} & \phi_{[2,0, k-1]} \\
\lambda_{n-1}^{n} \phi_{n-1} & \lambda_{n-2}^{n-2} \phi_{n-1} & \lambda_{n-1}^{n-3} \varphi_{n-1} & \ldots & \lambda_{n-1} \varphi_{n-1} & \phi_{n-1} \\
\lambda_{n}^{n} \phi_{n} & \lambda_{n}^{n-2} \phi_{n} & \lambda_{n}^{n-3} \varphi_{n} & \ldots & \lambda_{n} \varphi_{n} & \phi_{n}
\end{array}\right|,
\end{aligned}
$$




$$
W_{21}^{\prime}=\left|\begin{array}{cccccc}
\phi_{[1, n-1,1]} & \varphi_{[1, n-2,1]} & \phi_{[1, n-3,1]} & \ldots & \phi_{[1,1,1]} & \varphi_{[1,0,1]} \\
\phi_{[2, n-1,1]} & \varphi_{[2, n-2,1]} & \phi_{[2, n-3,1]} & \ldots & \phi_{[2,1,1]} & \varphi_{[2,0,1]} \\
\vdots & \vdots & \vdots & \vdots & \vdots & \vdots \\
\phi_{[1, n-1, k-1]} & \varphi_{[1, n-2, k-1]} & \phi_{[1, n-3, k-1]} & \ldots & \phi_{[1,1, k-1]} & \varphi_{[1,0, k-1]} \\
\phi_{[2, n-1, k-1]} & \varphi_{[2, n-2, k-1]} & \phi_{[2, n-3, k-1]} & \ldots & \phi_{[2,1, k-1]} & \varphi_{[2,0, k-1]} \\
\lambda_{n-1}^{n-1} \phi_{n} & \lambda_{n-1}^{n-2} \varphi_{n} & \lambda_{n-1}^{n-3} \phi_{n} & \ldots & \lambda_{n-1} \phi_{n} & \varphi_{n-1} \\
\lambda_{n}^{n-1} \phi_{n} & \lambda_{n}^{n-2} \varphi_{n} & \lambda_{n}^{n-3} \phi_{n} & \ldots & \lambda_{n} \phi_{n} & \varphi_{n}
\end{array}\right|,
$$

When $n=2 k+1$,

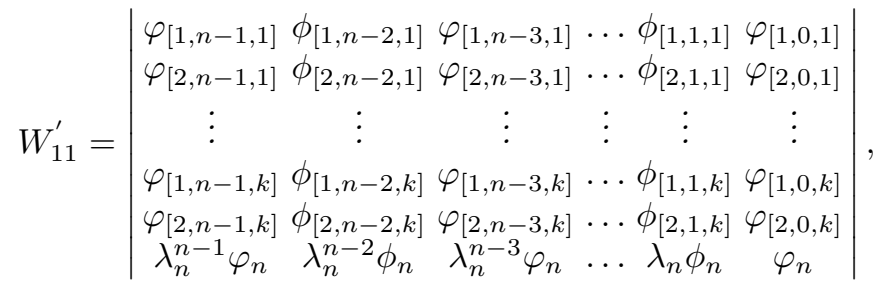

$$
\begin{aligned}
& W_{12}^{\prime}=\left|\begin{array}{cccccc}
\phi_{[1, n, 1]} & \phi_{[1, n-2,1]} & \varphi_{[1, n-3,1]} & \ldots & \phi_{[1,1,1]} & \varphi_{[1,0,1]} \\
\phi_{[2, n, 1]} & \phi_{[2, n-2,1]} & \varphi_{[2, n-3,1]} & \ldots & \phi_{[2,1,1]]} & \varphi_{[2,0,1]} \\
\vdots & \vdots & \vdots & \vdots & \vdots & \vdots \\
\phi_{[1, n, k]} & \phi_{[1, n-2, k]} & \varphi_{[1, n-3, k]} & \ldots & \phi_{[1,1, k]} & \varphi_{[1,0, k]} \\
\phi_{[2, n, k]} & \phi_{[2, n-2, k]} & \varphi_{[2, n-3, k]} & \ldots & \phi_{[2,1, k]]} & \varphi_{[2,0, k]} \\
\lambda_{n}^{n} \phi_{n} & \lambda_{n}^{n-2} \phi_{n} & \lambda_{n}^{n-3} \varphi_{n} & \ldots & \lambda_{n} \phi_{n} & \varphi_{n}
\end{array}\right|, \\
& W_{21}^{\prime}=\left|\begin{array}{cccccc}
\phi_{[1, n-1,1]} & \varphi_{[1, n-2,1]} & \phi_{[1, n-3,1]} & \ldots & \varphi_{[1,1,1]} & \phi_{[1,0,1]} \\
\phi_{[2, n-1,1]} & \varphi_{[2, n-2,1]} & \phi_{[2, n-3,1]} & \ldots & \varphi_{[2,1,1]} & \phi_{[2,0,1]} \\
\vdots & \vdots & \vdots & \vdots & \vdots & \vdots \\
\phi_{[1, n-1, k]} & \varphi_{[1, n-2, k]} & \phi_{[1, n-3, k]} & \ldots & \varphi_{[1,1, k]} & \phi_{[1,0, k]} \\
\phi_{[2, n-1, k]} & \varphi_{[2, n-2, k]} & \phi_{[2, n-3, k]} & \ldots & \varphi_{[2,1, k]} & \phi_{[2,0, k]} \\
\lambda_{n}^{n-1} \phi_{n} & \lambda_{n}^{n-2} \varphi_{n} & \lambda_{n}^{n-3} \phi_{n} & \ldots & \lambda_{n} \varphi_{n} & \phi_{n}
\end{array}\right| .
\end{aligned}
$$

Proof Define the function $\Psi[i, j, k]$ as follows

$$
\lambda_{i}^{j} \Phi=\Psi[i, j, 0]+\Psi[i, j, 1] \varepsilon+\Psi[i, j, 2] \varepsilon^{2}+\cdots+\Psi[i, j, k] \varepsilon^{k}+\cdots
$$

where $\varepsilon$ is a small parameter, and $\Psi[i, j, k]$ is the coefficient of $\varepsilon^{k}$ when expanding $\lambda^{j} \Psi$ at $\lambda=$ $\lambda_{i}(i, j=1,2, \ldots, N)$ via the Taylor expansion. $\Psi[i, j, k]=\left(\begin{array}{c}\phi[i, j, k] \\ \psi[i, j, k]\end{array}\right)=\frac{1}{k !} \frac{\partial^{k}}{\partial \varepsilon^{k}}\left[\left(\lambda_{i}+\varepsilon\right)^{j} \Psi\left(\lambda_{i}+\varepsilon\right)\right]$.

Combining the Taylor expansion and formula (24), we can derive the $k-1$ order rogue waves on the double-periodic background when $N=2 k$.

Starting from formula (24), set $\lambda_{1}=\frac{1}{2} \sqrt{2 a-c^{2}}-\frac{1}{2} i c+\varepsilon_{1}, \lambda_{2}=-\frac{1}{2} \sqrt{2 a-c^{2}}-\frac{1}{2} i c+\varepsilon_{1}$ and do the first order Taylor expansion in all the elements of the first and second row with respect to $\varepsilon_{1}$. Extract $\varepsilon_{1}$ in the first and second row, then take $\varepsilon_{1} \rightarrow 0$;

Take $\lambda_{2 k-3}=\frac{1}{2} \sqrt{2 a-c^{2}}-\frac{1}{2} i c+\varepsilon_{2 k-3}, \lambda_{2 k-2}=-\frac{1}{2} \sqrt{2 a-c^{2}}-\frac{1}{2} i c+\varepsilon_{2 k-3}, k \geq 3$ and do the $(k-1)$ order Taylor expansion in all the elements of the $(2 k-3)$-th and $(2 k-2)$-th row with 
respect to $\varepsilon_{2 k-3}$. Subtract the first, third,..., $(2 k-5)$-th row from the $(2 k-3)$-th row, subtract the second, fourth,..., $(2 k-4)$-th row from the $(2 k-2)$-th row. Extract $\varepsilon_{2 k-3}^{k-1}$ in the $(2 k-3)$-th row and $(2 k-2)$-th row, then take $\varepsilon_{2 k-3} \rightarrow 0$;

Remain all the elements of the $(2 k-1)$-th and $2 k$-th row unchanged, where $\varepsilon_{k}^{m}(m=1,2, \ldots, N)$ is the $m$-th power of the real constant $\varepsilon_{k}$.

Let $N=2 k, \lambda_{1}=\frac{1}{2} \sqrt{2 a-c^{2}}-\frac{1}{2} i c, \lambda_{2}=-\frac{1}{2} \sqrt{2 a-c^{2}}-\frac{1}{2} i c, \lambda_{2 k-1}=i \beta_{2 k-1}$ and $\lambda_{2 k}=i \beta_{2 k}$. Based on the above procedures, the $k-1$ order rogue waves on the double-periodic background for (2) are obtained.

Same with the procedure of $N=2 k+1$, and remain all the elements of the $(2 k+1)$-th row unchanged, we can derive the $k$ order rogue waves on the periodic background in terms of the determinant expression for Eq. 2 when $N=2 k+1$.

\subsection{Rogue waves on the periodic background}

The higher order rogue waves on periodic background can be generated by odd order generalized DT. For $N=3, \lambda_{1}=\frac{1}{2} \sqrt{2 a-c^{2}}-\frac{1}{2} i c, \lambda_{2}=-\frac{1}{2} \sqrt{2 a-c^{2}}-\frac{1}{2} i c$ and $\lambda_{3}=i \beta_{3}$. Here, set $a=1, c=1$ for convenience, the first-order rogue waves $q_{3}$ on the periodic background for Eq. (2) can be obtained. The patterns of $q_{3}$ are displayed in Fig $7(\mathrm{a})$ and Fig.7(b). For $\beta_{3}>0$, the rogue wave pattern locates on the area where the periodic pattern reaches its amplitude. However, for $\beta_{3}<0$, the rogue wave pattern locates in the middle of two amplitude trajectories of the periodic pattern, which looks like that the rogue wave is generated by the interaction of two waves of the periodic pattern.

For $N=5, \lambda_{1}=\frac{1}{2} \sqrt{2 a-c^{2}}-\frac{1}{2} i c, \lambda_{2}=-\frac{1}{2} \sqrt{2 a-c^{2}}-\frac{1}{2} i c$ and $\lambda_{5}=i \beta_{5}$, the second-order rogue waves on the periodic background for Eq. (2) was shown in Fig.8. The second-order rogue waves have a high amplitude peak on the center distributed with some lower peaks and four caves. Same with the case $N=3$, for $\beta_{5}>0$, the rogue wave pattern locates on the area where the periodic pattern reaches its amplitude (see Fig $8(\mathrm{a})$. For $\beta_{5}<0$, the rogue wave pattern locates in the middle of two amplitude trajectories of the periodic pattern (see Fig, $8(\mathrm{~b})$ ). And the periodic background can influence the peak value of the rogue wave.

\subsection{Rogue waves on the double-periodic background}

The rogue waves on the dual-periodic background are complicated, only the first-order solution is given in this section. When $N=4$, let $\lambda_{1}=\frac{1}{2} \sqrt{2 a-c^{2}}-\frac{1}{2} i c, \lambda_{2}=-\frac{1}{2} \sqrt{2 a-c^{2}}-\frac{1}{2} i c$, $\lambda_{3}=i \beta_{3}$ and $\lambda_{4}=i \beta_{4}$. We can obtain the first-order rogue waves $q_{4}$ on the double-periodic background for Eq. (2). It shows rogue waves with a double-periodic background, which is similar to the Jacobi elliptic function-type seed solution. The selection of parameters has an effect on the amplitude of the double-periodic background and the amplitude of the rogue waves. And the interesting thing is that there are two peaks on the double-periodic background when take $a=1, c=1, \beta_{3}=0.1, \beta_{4}=\frac{\sqrt{2}}{2}$ (see Fig 9(a). And there are four peaks on the double-periodic background when we take $a=1, c=\frac{1}{2}, \beta_{3}=0.1, \beta_{4}=\frac{\sqrt{2}}{2}$ (see Fig 9(b). Significantly, when $\beta_{3}=-\beta_{4}$, the rogue waves on the double-periodic background will convert to the rogue waves on 

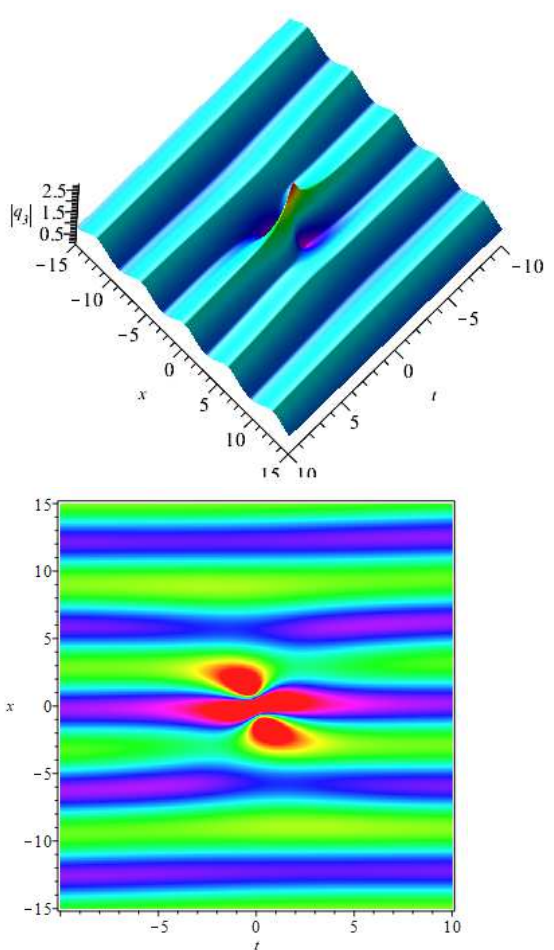

(a)
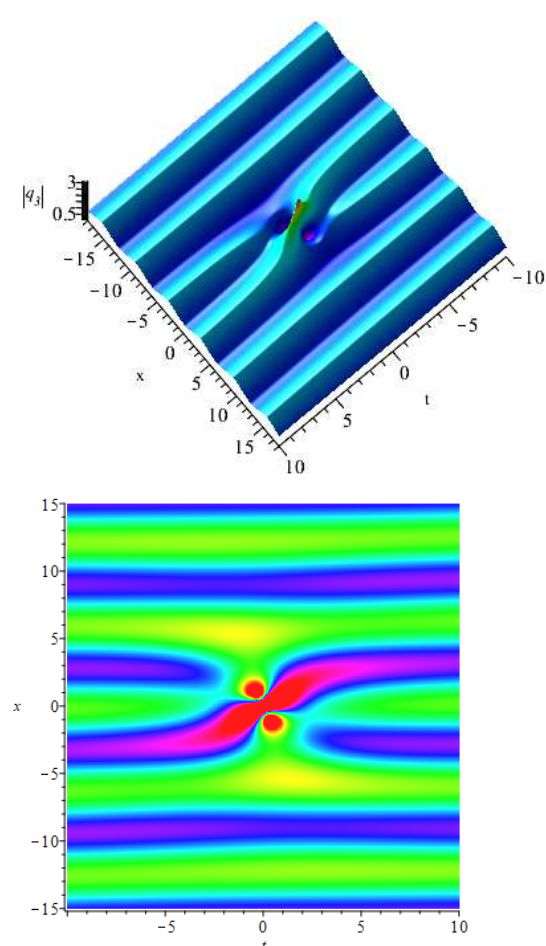

(b)

Fig. 7: One-order rogue waves on the periodic background: (a) $a=1, c=1, \beta_{3}=\frac{1}{10}$; (b) $a=1$, $c=1, \beta_{3}=-\frac{1}{10}$.

the plane wave (see Fig $9(\mathrm{c})$ ).

\section{Conclusion}

In summary, the breathers and rogue waves on the double-periodic background for Eq.22 have been constructed, which are first generated by plane wave seed solution. For the $N$-fold DT formula, when $N=4, \lambda_{2}= \pm \lambda_{1}^{*}, \lambda_{3}$ and $\lambda_{4}$ are pure imaginary numbers, the breathers on the double-periodic background have been obtained. We have found the breathers on the double-periodic background more easily observed in the $t$ direction by dynamics analysis.

Based on the $N$-fold DT formula, taking special eigenvalue which makes the corresponding eigenfunction degenerate. Let $\lambda_{N-1}=i \beta_{N-1}, \lambda_{N}=i \beta_{N}$, the even order generalized DT has been constructed by Taylor expansion. Then the higher order rogue waves on double-periodic background are constructed by even order generalized DT. For the case of $N=4, \lambda_{3}=i \beta_{3}$ and $\lambda_{4}=i \beta_{4}$. The two peak and four peak rogue waves on the double-periodic background for (2) have been obtained, which are new solutions. The selection of parameters has an effect on the amplitude of the rogue peaks and double-periodic background, and also influences the numbers of rogue peaks. Another interesting effect has been when $\beta_{3}=-\beta_{4}$, the rogue waves on the double-periodic background will convert to the classical rogue waves on the plane wave. 

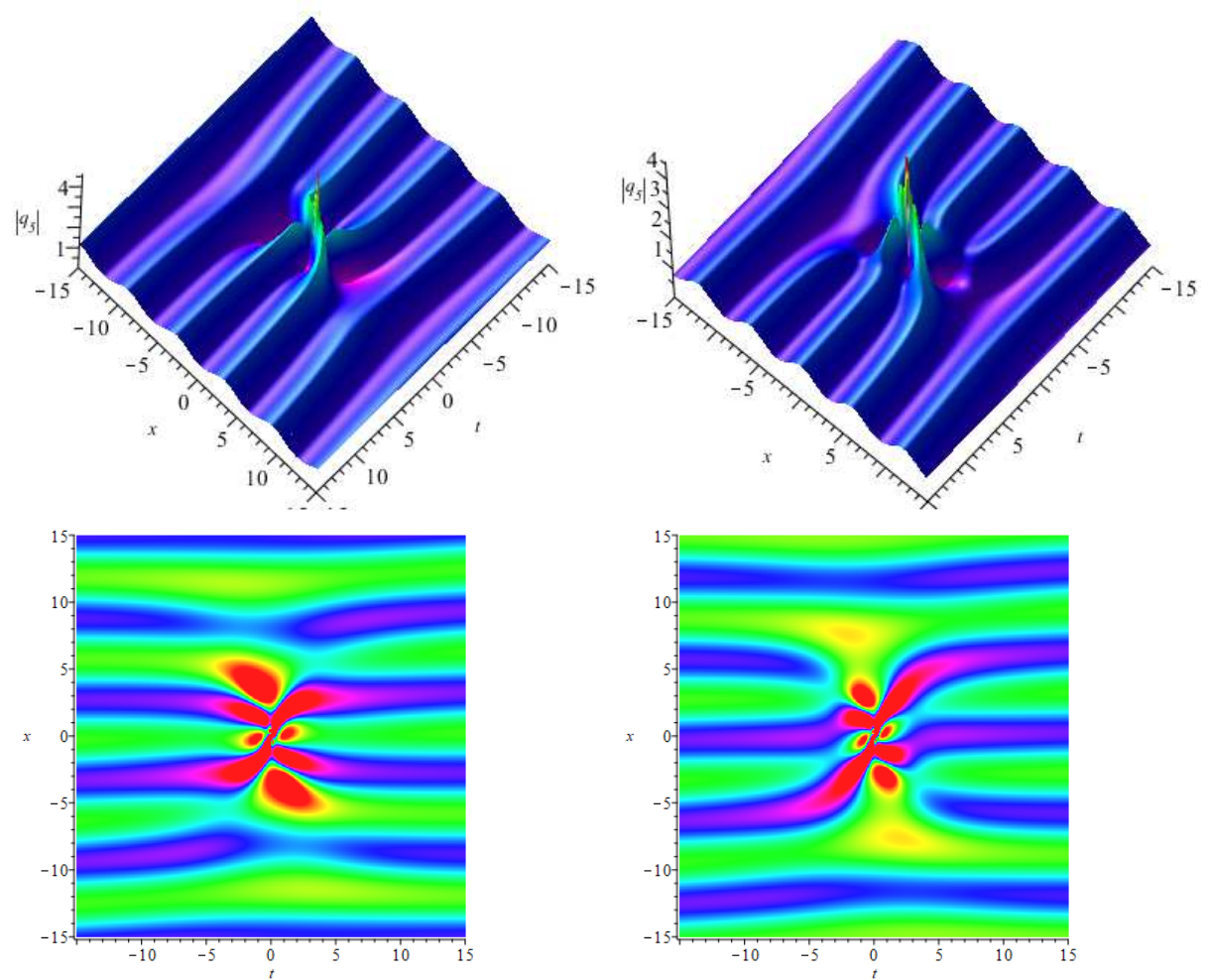

(a)

(b)

Fig. 8: Second-order rogue waves on the periodic background: (a) $a=1, c=1, \beta_{5}=\frac{1}{10}$; (b) $a=1, c=1, \beta_{5}=-\frac{1}{10}$.

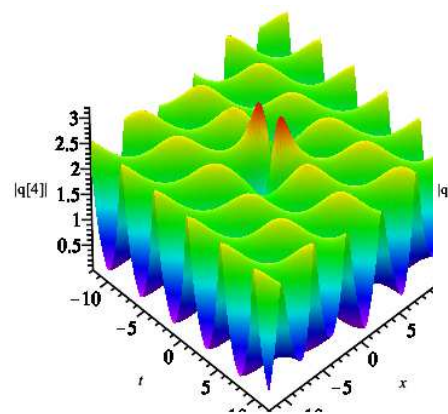

(a)

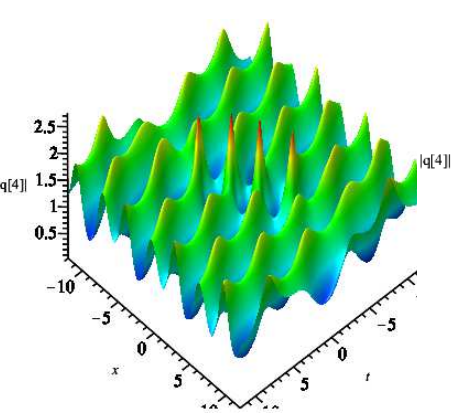

(b)

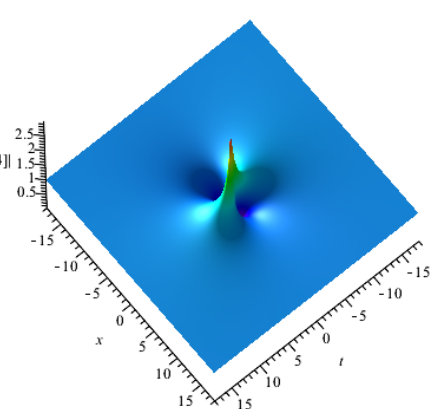

(c)

Fig. 9: Rogue waves on the double-periodic background: (a) $a=1, c=1, \beta_{3}=0.1, \beta_{4}=\frac{\sqrt{2}}{2}$; (b) $a=1, c=\frac{1}{2}, \beta_{3}=0.1, \beta_{4}=\frac{\sqrt{2}}{2} ;$ (c) $a=1, c=1, \beta_{3}=\frac{\sqrt{2}}{2}, \beta_{4}=-\frac{\sqrt{2}}{2}$.

Similar, for DT formula (24), when $N=3, \lambda_{2}= \pm \lambda_{1}^{*}$, and $\lambda_{3}$ is a pure imaginary number, the breathers on the periodic background have been obtained. The amplitude of the periodic background and period of the breathers are directly influenced by the parameters. There are 
some new phenomenons: the crest of the Ma breathers is cut and the phase shift occurs at the center of the general breathers with the perturbation of periodic background.

For the odd order generalized DT formula (42), when $N=3, N=5$. The first and second order rogue waves on the periodic background for Eq.(2) have been obtained. When $\beta_{N}>0$, the rogue wave patterns are located in the area where the periodic pattern reaches its amplitude. However, when $\beta_{N}<0$, the rogue wave patterns locate in the middle of two amplitude trajectories of the periodic pattern, which looks like that the rogue wave was generated by the interaction of two waves of the periodic pattern. The higher-order rogue waves have a high amplitude peak on the center distributed with some lower peaks and even numbers of caves. And the periodic background can influence the peak value of the rogue wave.

The soliton solution, rational solution, periodic solution, double-periodic solution, breathers and rogue wave for Eq. (2) also have been constructed in this work. Therefore, the addition of nonlocal terms in nonlinear equation greatly enrich the structure of the solutions.

\section{Acknowledge}

The authors would like to thank Lou Senyue, Fan Engui, Yan Zhenya, Peng Weiqi and Wang Minmin for their valuable comments and suggestions. This work was supported by Global Change Research Program of China (No.2015CB953904), National Natural Science Foundation of China (No.11675054) and Science and Technology Commission of Shanghai Municipality (No.18dz2271000).

\section{Declarations}

\section{Conflict of interests}

The authors declare that there is no conflict of interests regarding the publication of this paper.

\section{Data availability statement}

All date generated or analysed during this study are included in this published article.

\section{References}

1. Abrarov R.M., Christiansen P.L., Darmanyan S.A., Scott A.C., Soerensen M.P.: Soliton propagation in three coupled nonlinear Schrödinger equations, Phys. Lett. A. 171, 298-302 (1992)

2. Zhang, G.Q., Yan, Z.Y, Chen, Y.: Novel higher-order rational solitons and dynamics of the defocusing integrable nonlocal nonlinear Schrödinger equation via the determinants. Appl. Math. Lett. 69, 113-120 (2017)

3. Ling, L.M., Feng, B.F., Zhu, Z.N.: General soliton solutions to a coupled Fokas-Lenells equation, Nonlinear Anal. Real. 40, 185-214 (2018)

4. Yang, J.K.: General N-solitons and their dynamics in several nonlocal nonlinear Schrödinger equations, Phys. Lett. A 383(4), 328-337 (2019)

5. Ma, Y.C.: The perturbed plane-wave solutions of the cubic Schrödinger equation, Stud. Appl. Math. 60, 43-58 (1979)

6. Akhmediev, N.N., Korneev, V.I.: Modulation instability and periodic solutions of the nonlinear Schrödinger equation. Theor. Math. Phys. 69, 1080-1093 (1986)

7. Dudley, J.M., Dias, F., Erkintalo, M., Genty, G.: Instabilities, breathers and rogue waves in optics, Nat. Photonics 8, 755-764 (2014)

8. Draper, L.: Freak ocean waves, Weather 21, 2-4 (1966) 
9. Solli, D.R., Ropers, C., Koonath, P., Jalali, B.: Rogue waves and rational solutions of the nonlinear Schrödinger equation, Nature 450, 1054 (2007)

10. Bludov, Y.V., Konotop, V.V., Akhmediev, N.: Matter rogue waves, Phys. Rev. A 80, 033610 (2009)

11. Yan, Z.Y.: Vector financial rogue waves, Phys. Lett. A 375 4274-4279 (2011)

12. C. Z. Li, J. S. He: Darboux transformation and positons of the inhomogeneous Hirota and the Maxwell-Bloch equation, Sci. China Phys. Mech. 57, 898-907 (2014)

13. Zhou, H.J., Li, C.Z.: A weakly coupled Hirota equation and its rogue waves, Mod. Phys. Lett. A 34, 1950179 (2019)

14. Jin, X.W., Lin, J.: Rogue wave, interaction solutions to the KMM system J. Magn. Magn. Mater. 502, 166590 (2020)

15. Peng, W.Q., Pu, J.C., Chen, Y.: PINN deep learning for the Chen-Lee-Liu equation: Rogue wave on the periodic background, arXiv:2105.13027v1 (2021)

16. Kundu, A.: Two-fold integrable hierarchy of nonholonomic deformation of the derivative nonlinear Schrödinger and the Lenells-Fokas equation, J. Math. Phys. 51, 022901 (2010)

17. Xu, S.W., He, J.S., Wang, L.H.: The Darboux transformation of the derivative nonlinear Schrödinger equation, J. Phys. A-Math. Theor. 44, 6629-6636 (2011)

18. Zhang, Y.S., Guo, L.J., Xu, S.W., Wu, Z.W., He, J.S.: The hierarchy of higher order solutions of the derivative nonlinear Schrödinger equation, Commun. Nonlinear Sci. 19, 1706-1722 (2014)

19. Xu, T., Chen, Y.: Mixed interactions of localized waves in the three-component coupled derivative nonlinear Schrödinger equations, Nonlinear Dyn. 92, 2133-2142 (2018)

20. Mjфlhus, E.: On the modulational instability of hydromagnetic waves parallel to the magnetic field, J. Plasma Phys. 16, 321-334 (1976)

21. Lakhina, G.S., Sharma, A.S., Buchner, J.: International workshops on nonlinear waves and chaos in space plasmas-preface, Nonlinear Proc. Geoph. 11(2), 181-181 (2004)

22. Ruderman, M.S.: DNLS equation for large-amplitude solitons propagating in an arbitrary direction in a high- $\beta$ hall plasma, J. Plasma Phys. 67, 271-276 (2002)

23. Shan, S.A., El-Tantawy, S.A.: The impact of positrons beam on the propagation of super freak waves in electron-positron-ion plasmas, Phys. Plasmas 23(7), 072112 (2016)

24. Tzoar, N., Jain, M.: Self-phase modulation in long-geometry optical waveguide, Phys. Rev. A. 23, 1266-1270 (1981)

25. Anderson, D., Lisak, M.: Nonlinear asymmetric self-phase modulation and self-steepening of pulses in long Optical Waveguides, Phys. Rev. A 27, 1393-1398 (1983)

26. Govind, P.A.: Nonlinear fibers optics 3rd edn, New York: Adademic (2001)

27. Zeng, Y.: New factorization of the Kaup-Newell hierarchy, Physica D. 73, 171-188 (1994)

28. Zhou, Z.X.: Parameters of darboux transformation for reduced akns, kaup-newell and pcf systems, Chinese Ann. Math. B 20, 195-204 (1999)

29. Zhou, Z.X.: Darboux transformations and global solutions for a nonlocal derivative nonlinear Schrödinger equation, Commun. Nonlinear Sci. 62, 480-488 (2016)

30. Matveev, V.B., Salle, M.A.: Darboux transformations and solitons, Springer, Berlin-Heidelberg (1991)

31. Li, Y.S.: Soliton and integrable system, Shanghai Sci.-Tech. Edu., Publishing House, Shanghai (1991)

32. Gu, C.H.: Darboux transformation in soliton theory and its geometric applications, Shanghai Sci.-Tech. Edu., Publishing House, Shanghai (2005)

33. Gu, C.H., Hu, H.S., Zhou, Z.X.: Darboux Transformations in Integrable Systems: Theory and Their Applications (Berlin: Springer) 2005.

34. Xu, T., Li, H.j., Zhang, H.j., Li, M., Lan, S.: Darboux transformation and analytic solutions of the discrete PT-symmetric nonlocal nonlinear Schrödinger equation, Appl. Math. Lett. 63, 88-94 (2017)

35. Wang, M.M., Chen Y.: Dynamic behaviors of mixed localized solutions for the three-component coupled Fokas-Lenells system. Nonlinear Dyn. 98(3), 1781-1794 (2019)

36. Shi, Y., Shen, S.F., Zhao, S.L.: Solutions and connections of nonlocal derivative nonlinear Schrödinger equations, Nonlinear Dyn. 95, 1257-1267 (2019)

37. Meng, D.X., Li, K.Z.: Darboux transformation of the second-type nonlocal derivative nonlinear Schrödinger equation, Mod. Phys. Lett. B 33(10), 1950123 (2019)

38. Ablowitz, M.J., Musslimani, Z.H.: Integrable nonlocal nonlinear equations, Phys. Rev. Lett. 110, 064105 (2013)

39. Kedziora, D.J., Ankiewicz, A., Akhmediev, N.: Rogue waves and solitons on a cnoidal background, Eur. Phys. J-Spec. Top. 223(1), 43-62 (2014)

40. Chen, J.B., Pelinovsky, D.E.: Rogue periodic waves of the modified KdV equation, Nonlinearity 31, 1955-1980 (2018)

41. Sinthuja, N., Manikandan, K., Senthilvelan, M.: Rogue waves on the double-periodic background in Hirota equation, Eur. Phys. J. Plus. 136(3), 1-12 (2021)

42. Huang, X., Ling, L.M.: Soliton solutions for the nonlocal nonlinear Schrödinger equation. Eur. Phys. J. Plus 131, $148(2016)$ 
43. He, J.S., Tao, Y.S., Porsezian, K., Fokas, A.: Rogue wave management in an inhomogeneous Nonlinear Fibre with higher order effects, J. Nonlinear Math. Phys. 20, 407-419 (2013)

44. He, J.S., Charalampidis, E.G., Kevrekidis, P.G., Frantzeskakis, D.J.: Rogue waves in nonlinear Schrödinger models with variable coefficients: Application to Bose-Einstein condensates, Phys. Lett. A 378(56), 577-583 (2014)

45. Zhao, L.C., Ling, L.M., Qi, J.W., Yang, Z.Y., Yang, W.L.: Dynamics of rogue wave excitation pattern on stripe phase backgrounds in a two-component Bose-Einstein condensate, Commun. Nonlinear Sci. Numer. Simulat, 49, 39-47 (2017)

46. Liu, W., Zhang, Y.S., He, J.S.: Rogue wave on a periodic background for Kaup-Newell equation, Rom. Rep. Phys. 70, 106 (2018)

47. Ding, C.C., Gao, Y.T., Li, L. Q.: Breathers and rogue waves on the periodic background for the GerdjikovIvanov equation for the Alfvén waves in an astrophysical plasma, Chaos Soliton. Fract. 120, 259-265 (2019)

48. Randoux, S., Suret, P., Chabchoub, A., Kibler, B., El, G.: Nonlinear spectral analysis of Peregrine solitons observed in optics and in hydrodynamic experiments, Phys. Rev. E 98, 022219 (2018).

49. Chen, J.B., Pelinovsky, D.E., White, R.E.: Rogue waves on the double-periodic background in the focusing nonlinear Schrödinger equation, Phys. Rev. E 100, 052219 (2019)

50. Rao, J.G., Zhang, Y.S., Fokas, A.S., He, J.S.: Rogue waves of the nonlocal Davey-Stewartson I equation, Nonlinearity 31(9), 4090-4107 (2018)

51. Xue, B., Shen, J., Geng, X.G.: Breathers and breather-rogue waves on a periodic background for the derivative nonlinear Schrödinger equation, Phys. Scripta 95(5), 055216 (2020)

52. Liu, Y., Li, B.: Dynamics of solitons and breathers on a periodic waves background in the nonlocal Mel'nikov equation, Nonlinear Dyn. 100(4), 3717-3731 (2020)

53. Zhang, H.Q., Chen, F., Pei, Z.J.: Rogue waves of the fifth-order Ito equation on the general periodic travelling wave solutions background, Nonlinear Dyn. 103, 1023-1033 (2021)

54. Fan, E.G.: A Liouville integrable Hamiltonian system associated with a generalized Kaup-Newell spectral problem, Physica A. 301, 105-113 (2001)

55. Ma, W.X., Zhou, R.: A coupled AKNS-Kaup-Newell soliton hierarchy, J. Math. Phys. 40(9), 4419-4428 (1999) 